\title{
PU.1 drives specification of pluripotent stem cell-derived endothelial cells to LSEC-like cells
}

\author{
Jonathan De Smedt [1]', Elise Anne van Os², Irene Talon', Sreya Ghosh', Burak Toprakhisar', \\ Rodrigo Furtado Madeiro Da Costa', Samantha Zaunz', Marta Aguirre Vazquez', Ruben Boon (10,4, Pieter Baatsen', \\ Ayla Smout ${ }^{2}$, Stefaan Verhulst $\mathbb{1}^{2}$, Leo A. van Grunsven $\mathbb{B}^{2}$ and Catherine M. Verfaillie $\mathbb{B}^{1}$
}

\begin{abstract}
To date, there is no representative in vitro model for liver sinusoidal endothelial cells (LSECS), as primary LSECs dedifferentiate very fast in culture and no combination of cytokines or growth factors can induce an LSEC fate in (pluripotent stem cell (PSC)-derived) endothelial cells (ECs). Furthermore, the transcriptional programmes driving an LSEC fate have not yet been described. Here, we first present a computational workflow (CenTFinder) that can identify transcription factors (TFs) that are crucial for modulating pathways involved in cell lineage specification. Using CenTFinder, we identified several novel LSEC-specific protein markers, such as FCN2 and FCN3, which were validated by analysis of previously published single-cell RNAseq data. We also identified PU.1 (encoded by the SPI1 gene) as a major regulator of LSEC-specific immune functions. We show that SPI1 overexpression (combined with the general EC TF ETV2) in human PSCS induces ECs with an LSEC-like phenotype. The ETV2-SPI1-ECs display increased expression of LSEC markers, such as CD32B and MRC1, as well as several of the proposed novel markers. More importantly, ETV2SPI1-ECs acquire LSEC functions, including uptake of FSA-FITC, as well as labelled IgG. In conclusion, we present the CenTFinder computational tool to identify key regulatory TFs within specific pathways, in this work pathways of lineage specification, and we demonstrate its use by the identification and validation of PU.1 as a master regulator for LSEC fating.
\end{abstract}

\section{Introduction}

Liver sinusoidal endothelial cells (LSECs) are highly specialised endothelial cells (ECs) lining the sinusoidal capillaries of the liver, where they reside along the space of Disse separating them from hepatocytes. LSECs have five main functions. First, LSEC fenestrations actively regulate the flow of macromolecules, such as lipids and chylomicron remnants towards the hepatocytes ${ }^{1,2}$. Second, LSECs endocytose larger lipid complexes, soluble

\footnotetext{
Correspondence: Jonathan De Smedt (jonathan.desmedt@kuleuven.be) or Catherine M. Verfaillie (catherine.verfaillie@kuleuven.be)

${ }^{1}$ Department of Development and Regeneration, Stem Cell Institute, KU Leuven, Leuven, Belgium

${ }^{2}$ Liver Cell Biology research group, Vrije Universiteit Brussel (VUB), Brussels, Belgium

Full list of author information is available at the end of the article

These authors contributed equally: Leo A. van Grunsven, Catherine M. Verfaillie Edited by I. Amelio
}

macromolecules, hyaluronan, and glycosylation end products via the mannose receptor (MR or CD206), CD32B, stabilin-1 (STAB1), and stabilin-2 (STAB2) ${ }^{3,4}$. Third, in response to hepatic injury, LSECs regulate liver regeneration by releasing hepatocyte growth factor, WNT2, and angiopoietin- $2^{5,6}$. Fourth, LSECs can clear a multitude of viruses $^{7-12}$. Furthermore, LSECs clear bacterial compounds via the MR, STAB1, and STAB2 receptors. LSECs also express Toll-like receptors, as well as several proteins involved in inflammasome formation (such as NLRP-1, NLRP-3, and AIM2) ${ }^{13}$. Expression of CD32B allows uptake of IgG immunocomplexes, while STAB2 is involved in endocytosis of Gram-positive and -negative bacteria. Finally, LSECs have a major role in adaptive immunity. LSECs are antigen-presenting cells through MR-mediated antigen uptake and expression of MHC-I ${ }^{14}$. Upon antigen presentation by MHC-I, LSECs can activate

\section{(c) The Author(s) 2021}

(c) (i) Open Access This article is licensed under a Creative Commons Attribution 4.0 International License, which permits use, sharing, adaptation, distribution and reproduction cc) in any medium or format, as long as you give appropriate credit to the original author(s) and the source, provide a link to the Creative Commons license, and indicate if changes were made. The images or other third party material in this article are included in the article's Creative Commons license, unless indicated otherwise in a credit line to the material. If material is not included in the article's Creative Commons license and your intended use is not permitted by statutory regulation or exceeds the permitted use, you will need to obtain permission directly from the copyright holder. To view a copy of this license, visit http://creativecommons.org/licenses/by/4.0/. 
CD8 + T cells. In addition, LSECs are the main cells in the liver responsible for the conversion of $\mathrm{CD} 4+\mathrm{T}$ cells into CD4+ CD25+ Foxp3+ regulatory T cells ${ }^{15}$.

In pathological conditions, LSECs become dysfunctional and play a role in non-alcoholic fatty liver disease and fibrosis ${ }^{16-19}$. In liver fibrosis, LSECs dedifferentiate, lose their fenestrations, and deposit a basement membrane (a process called capillarisation). As a result, when chronic fibrosis or cirrhosis ensues, lack of blood flow towards hepatocytes aggravates hepatotoxicity and inflammation, leading to portal hypertension ${ }^{20}$.

It should therefore be clear that LSECs, that play important roles in crosstalk with other (non-) parenchymal liver cell types in health and disease, should be included in liver co-culture models. However, primary LSECs very quickly lose their characteristics (e.g., fenestrae and endocytic capacity) and undergo apoptosis within 4-6 days of in vitro culture $^{21-23}$. Furthermore, limited availability of human primary LSECs is a major obstacle in the progression of LSEC research. A few immortalised LSEC lines exist, such as SK-HEP-1, that retain some LSEC characteristics ${ }^{24}$. However, SK-HEP-1 cells do not take up FITC-ALB ${ }^{24}$ and immortalised lines may not respond similarly in co-culture and disease models as primary LSECs do.

A number of protocols have been described to differentiate PSCs towards ECs ${ }^{25-27}$. However, only a few studies have attempted to differentiate PSCs into LSECs, either through culturing PSC-derived ECs in hypoxic conditions with a TGF $\beta$ inhibitor ${ }^{28}$, or the combination of CAMP and a TGF $\beta$ inhibitor ${ }^{29}$. Classically, differentiation protocols for a given cell type deploy sequential growth factor and cytokine cocktails to mimic in vivo differentiation. However, relatively little is known regarding LSEC development and, hence, signals required for LSEC differentiation in vitro. It has been hypothesised that LSECs derive from the septum transversum mesenchyme ${ }^{30-32}$. However, recent lineage tracing experiments suggest that a large portion of the liver vasculature may derive from the sinus venosus endocardium ${ }^{33}$. It is yet to be elucidated, though, whether the endocardium contributes to LSECs rather than macrovascular ECs. As an alternative, transcription factors (TFs) have been overexpressed to guide differentiation of PSCs towards cell types of all germ layers ${ }^{34-37}$. However, the transcriptional programmes or TFs that drive LSEC development are not well known.

Here, we designed a novel integrative meta-analysis pipeline (available as the CenTFinder $\mathrm{R}$ package) to facilitate analysis of published EC microarray data, and identified pathways and TFs that might be crucial in fating LSECs. We combined several complementary analysis methods, including gene co-expression analysis (using weighted gene correlation network analysis (WGCNA) ${ }^{38}$ ), TF motif binding enrichment analysis (using Rcis$\operatorname{Target}^{39}$ ), and differential expression analysis on bulk
RNAseq data (using DeSeq2 $2^{40}$ ), and identified several candidate TFs that may play a role in LSEC differentiation. As SPI1, encoding for PU.1, was identified as one of the top candidate TFs central to transcriptional networks for LSECs, we overexpressed SPI1 in PSCs, and demonstrated that this drives specification of PSC progeny to an LSEC-like phenotype.

\section{Materials and methods \\ Microarray selection and retrieval}

We defined query keywords, i.e., 'EC AND Homo sapiens' and 'LSEC AND Homo sapiens', to construct URLs with base https://www.ebi.ac.uk/arrayexpress/xml/ v3/experiments?keywords $=$ to query the ArrayExpress database, using the RCurl R package. ArrayExpress study accession codes were extracted from the HTTP responses. Subsequently, the respective SDRF files were downloaded to select relevant samples within each study. Only Affymetrix samples that were of human non-cancerous origin, and that were biotin-labelled were kept for analysis. Subsequently, new URLs were constructed to download the selected CEL files.

\section{Microarray raw analysis}

Platform information was extracted from all downloaded CEL files using the affyio $\mathrm{R}$ package. Microarray platforms with only one downloaded CEL file were excluded from analysis. Subsequently, for each platform type, the respective CEL files were RMA normalised, using the oligo $\mathrm{R}$ package. Probe intensity values were aggregated per gene by their geometric mean.

\section{Platform selection and platform effect removal}

As microarray platforms differed in the number of genes for which probes were coated, we weighed the benefits of analysing more samples and hence fewer genes in common or analysing fewer samples with more genes in common. For standard analyses, we selected as a rule of thumb those platforms for which the product of the number of samples and the number of common genes was maximised. However, the LSEC arrays of platform type HG-U219 contained probes for a slightly smaller than optimal number of genes. Nevertheless, as these were the only retrieved LSEC arrays, we included these arrays as well and, hence, the downstream analysis was performed with 279 EC arrays and 16,248 genes. Subsequently, we applied the Combat algorithm of the sva $\mathrm{R}$ package to remove platform and batch confounding effects.

\section{Gene filtering}

We excluded TFs that did not change by at least twofold across all EC microarray samples, as such minimal expression changes are not likely to be biologically meaningful. A list of human TFs was downloaded from 
http://humantfs.ccbr.utoronto.ca. Furthermore, to save on computational time, we restricted the number of genes that were not TFs to the 7,551 most variable ones $(\log 2$ fold change standard deviation $>0.5961016$ ). The total number of genes for downstream analysis was thus set to 9,000 .

\section{Weighted gene correlation network analysis}

WGCNA was performed using the WGCNA R package. We constructed a signed network and tested softthresholding powers ranging from 1 to 30. A cluster dendrogram was created based on the topological overlap matrix with a minimum cluster size of 30 . Subsequently, modules with a dissimilarity $<0.3$ were merged.

\section{Gene Ontology and KEGG pathway enrichment analysis}

Gene Ontology (GO) enrichment was calculated for all identified WGCNA modules, using Fisher's exact test from the topGO R package. The top $15 \mathrm{GO}$ terms were retained for each GO category (i.e., biological process, cellular component, and molecular function).

\section{RcisTarget analysis}

We imported motif rankings from the 'h19-tss-centred10kb-7species.mc9nr' database (https://resources.aertslab. org/cistarget/). Overrepresented DNA-binding motifs were identified within each of the WGCNA modules, using the RcisTarget $\mathrm{R}$ package according to the creators' guidelines.

\section{Bulk RNA-sequencing analysis \\ Sample acquisition}

PSCs, wherein the coding region of the TF ETV2 (controlled by a TET-ON system) was recombined in the AAVS1 locus (as described in Ordovas et al. $^{41}$ ), were differentiated until day 10 . RNA samples $(N=3)$ were prepared and sequenced on an Illumina NextSeq 500 platform by the VIB Nucleomics Core (KULeuven, Belgium). Three RNAseq samples annotated as primary human LSECs were obtained from ArrayExpress (EGEOD-40291 and E-GEOD-43984). However, only in one of these samples (E-GEOD-43984) LSEC-specific genes, FCGR2B, CLEC4M, STAB2, and CLEC4G, were expressed. The other samples were excluded from analysis.

\section{Read trimming}

Cutadapt was used to trim the adapter sequence from all reads. Bases with Phred-scores $<20$ were trimmed. Poly A $\{10\}$ tails were removed and reads shorter than 20 bases were removed.

\section{Genome index generation and read alignment}

The human genome fasta files (GRCh38.92) and GTF file were downloaded from Ensemble, and assembled into a genome index using STAR v020201. Subsequently,
STAR was used to align the filtered reads to the genome index. The $\mathrm{R}$ implementation of FeatureCounts (Rsubread) was used to generate raw read count matrices. Subsequently, we used the GTFtools Python package to calculate merged exon lengths, i.e., the union of all exons. Finally, we calculated the TPM matrix.

\section{Differential gene expression analysis}

The DESeq2 R package was used to calculate differential gene expression (Benjamini-Hochberg-corrected $p$ value $<0.05$ ). An absolute fold change of less than two was considered not biologically meaningful.

\section{Module gene set variant analysis}

We used the modules identified by WGCNA as gene sets in gene set variation analysis (GSVA), using the GSVA $\mathrm{R}$ package. Module gene sets were scored for each individual sample. Subsequently, mean GSVA scores were calculated per cell type to identify which modules were more prominently expressed for each cell type.

\section{Transcription factor ranking and visualisation}

TFs were considered for overexpression if they were higher expressed in LSECs compared to ECs derived from PSCs through overexpression of ETV2 (referred to as ETV2-ECs). In addition, TFs were only retained if their $k \mathrm{ME}$ centrality measure was $>0.5$, and if their respective clusters were more active (GSVA) in LSECs than in other ECs. Next, the TFs were ranked based on the fraction of genes in their respective modules they regulated (inferred by RcisTarget). The modules of interest were visualised using Cytoscape. Regulons of the modules of interest were extracted and reformatted into a dataframe with Source and Target column, suitable for Cytoscape network import. TFs of the modules of interest were visualised when differentially expressed, regardless of whether they were higher or lower expressed in LSECs. For visualisation purposes we only retained the 20 most differentially expressed downstream targets, as evaluated by bulk RNAseq data. TFs assigned by WGCNA to other modules than the modules of interest were given the colour of the module of interest if they had a high cluster centrality in the respective module of interest $(k M E>0.5)$. Node size was increased with increasing number of downstream targets.

\section{Single-cell RNA-sequencing analysis}

Fastq files from a recent human liver single-cell RNAsequencing study (GSE124395 $5^{42}$ ) were downloaded from the European Nucleotide Archive. Cellular barcode and UMI handling was done with UMI tools. We used Cutadapt to trim bases with Phred-score $<20$ and to remove reads shorter than 20 bases. Read quality was assessed with FastQC. STAR was used to create a human 
genome index based on the GRCh38.92 release of Ensemble. STAR was used as well for alignment. The count matrix was generated with UMI tools. Cell quality control was performed using the Scater and Seurat R packages. Briefly, we removed cells with $<2500$ UMIs or $<1500$ detected genes. Only cells with a mitochondrial read content $<15 \%$ were included for analysis. Only genes with a count higher than one in at least two cells were included for analysis. ENHANCE ${ }^{43}$ was used for imputation and denoising. The scran implementation (cyclone) of the method described by Scialdone et al. ${ }^{44}$. was used for cell cycle inference. SCENIC (with GRNBoost2 for ensemble learning) was used for detection of active gene regulatory networks $(\mathrm{GRNs})^{39}$. Seurat was used to correct for confounding variables, i.e., number of detected genes, number of total counts, cell cycle status, and percentage of mitochondrial counts.

The count matrix of a second study (GSE115469 ${ }^{45}$ ) was retrieved from the Gene Expression Omnibus. For each study, clusters were identified by Seurat and cell types were inferred by manually evaluating marker genes within the different clusters.

ECs were defined based on higher levels of $C D H 5$ and $K D R$ expression, and LSECs based on higher expression of CLEC4G, STAB1, and PECAM1. FCGR2B-low and FCGR2B-high LSEC subsets could be distinguished. Differential expression analysis was performed with a Wilcoxon rank-sum test followed by a Benjamini-Hochberg multiple test correction.

\section{Stem cell culture}

H9 embryonic stem cells (ESCs; WA09) were cultured in E8 Flex medium (cat. A2858501, ThermoFisher Scientific). Cells were passaged at $75 \%$ confluency using $0.1 \%$ EDTA in PBS (and tested for mycoplasma).

\section{Genome engineering of ESC lines for endothelial differentiation}

Two million H9 ESCs, engineered with an FRT and FRT3 site in the AAVS1 safe harbour locus ${ }^{41}$, were nucleoporated with a plasmid mix containing $2 \mu \mathrm{g}$ of pCAGGS-FLPe (MES4488, Open Biosystems; without the puromycin resistance gene) and $8 \mu \mathrm{g}$ of the donor plasmid containing the gene of interest. Donor plasmids also contained FRT and FRT3 sites in identical orientation, a promotorless puromycin cassette for gene trapping, and an inducible TETon system for overexpression of the gene of interest. The coding sequences of NM_014209.3 and of NM_001080547.1 were cloned into the donor plasmid template for overexpression of ETV2 and SPI1, respectively. ETV2 and SPI1 coding sequences were linked by a P2A autocleavage sequence. Nucleoporation and subsequent selection was performed, as described by Ordovas et al. $^{41}$.

\section{Differentiation of ESCs into endothelial cells}

H9 ESCs were passaged at a 1:6 ratio and grown for 1-2 days in E8 Flex medium to $40 \%$ confluency. On day 0 of endothelial differentiation, medium was changed to liver differentiation medium (LDM; composition as described in ref. ${ }^{37}$ ) and $5 \mu \mathrm{g} / \mathrm{ml}$ doxycycline. On day 2 of differentiation, cells were grown in LDM with $5 \mu \mathrm{g} / \mathrm{ml}$ doxycycline and $2 \%$ FBS. Cells were passaged on days 4,8 , and 12 in a 1:3 ratio. After day 12, no further passaging was performed. When 24-well plate formats were required, ECs were passaged on day 4 from 6-well plates into 24 -well plates ( 1 well into 10 wells, equivalent to a 1:2 split), and no further passaging was done until the time of read-out on day 12 of differentiation. To passage ECs, cells were washed with PBS and detached with StemPro $^{\mathrm{TM}}$ Accutase $^{\mathrm{TM}}$ Cell Dissociation Reagent (Gibco ${ }^{\mathrm{TM}}$, A1110501) for $35 \mathrm{~s}$ at $37^{\circ} \mathrm{C}$. Subsequently, accutase was removed, fresh medium added, and cells gently detached with a cell scraper. ECs derived from H9-ETV2 PSCs and H9-ETV2-SPI1 PSCs are further referred to as ETV2-ECs and ETV2-SPI1-ECs respectively. In the conditions where cells were supplemented with VEGFA (50 ng/ml; cat. 10020 , Peprotech), it was added to the culture medium as of day 6 of differentiation (Supplementary Fig. 1; $N=7$ for samples without VEGFA, $N=4$ for samples with VEGFA; statistical differences assessed by two-sided Student's $T$ test).

\section{Isolation of mouse LSECs and hepatic stellate cells}

Primary liver cells were isolated from 10- to 25-weekold BALB/c mice, $(N=3)$, as previously described ${ }^{46}$. LSECs and hepatic stellate cells (HSCs) were plated on collagen-coated wells in a 24-well plate $(200,000$ cells/ well) in 0\% FBS-DMEM for LSECs and 10\% FBS-DMEM for HSCs. All methods, experimental protocols and animal experimentation ethics were carried out in accordance with the approved guidelines of the Vrije Universiteit Brussel (VUB, Belgium) and according to European Guidelines for the Care and Use of Laboratory Animals. All animal experimentation protocols were approved by the Ethical Committee of Animal Experimentation of the Vrije Universiteit Brussel (VUB, Belgium; LA 12302 12, project 18-212-1).

\section{Lentiviral overexpression of SPI1, ERG, and FLI1}

H9-ETV2 PSCs were differentiated into ECs and passaged into 24-well plates on day 4 . On day 6, cells were transduced with lentiviral vectors encoding for SPI1, FLI1, and ERG1 (for ERG overexpressions: $N=2$; all other conditions: $N=3$ ) in the presence of $4 \mu \mathrm{g} / \mathrm{ml}$ polybrene (cat. TR-1003-G, Merck; Supplementary Fig. 2). Where $E T V 2$ overexpression was substituted for lentiviral overexpression of FLI1 and/or ERG, doxycycline was omitted from culture media from day 8 of differentiation onwards. 
Statistical differences were assessed by a two-sided Student's $T$ test.

\section{Reverse-transcriptase quantitative polymerase chain reaction}

Cells were lysed and RNA extracted with the GenElute $^{\mathrm{TM}}$ Mammalian RNA Extraction Kit (cat. RTN70, Sigma Aldrich) and reverse transcribed into cDNA, using SuperScript ${ }^{\circledR}$ III First-Strand Synthesis SuperMix (cat. 11752050, Invitrogen ${ }^{\mathrm{TM}}$ ), according to the manufacturer's protocol. Reverse-transcriptase quantitative polymerase chain reaction ( $\mathrm{RT}-\mathrm{qPCR}$ ) reactions were made using the Platinum ${ }^{\circledR}$ SYBR ${ }^{\circledR}$ Green RT-qPCR SuperMix-UDG kit (cat. 11733046, ThermoFisher Scientific). Reactions were denatured at $95^{\circ} \mathrm{C}$ for $20 \mathrm{~s}$, followed by 40 cycles of a $1 \mathrm{~s}$ denaturation step at $95^{\circ} \mathrm{C}$ and a $20 \mathrm{~s}$ annealing and elongation step at $60{ }^{\circ} \mathrm{C}$. Gene expression values were normalised for GAPDH, RPL19, RPS23, and EEF1A1. Primer sequences are listed in Supplementary Table 1.

\section{Immunostaining}

Culture medium was removed and cells (on coverslips) washed with PBS. Cells were fixed with $4 \%$ paraformaldehyde for $10 \mathrm{~min}$, washed with PBS and permeabilised with $0.2 \%$ Triton $\mathrm{X}-100$ in PBS. For CD32B staining, cells were not permeabilised. Blocking was performed using $5 \%$ donkey serum in $0.2 \%$ Triton X-100 in PBS. Primary antibodies (Supplementary Table 2) were diluted according to manufacturer's guidelines in DAKO Antibody Diluent (cat. S202230, Agilent). Cells were incubated with the primary antibody mixes overnight at $4{ }^{\circ} \mathrm{C}$, washed with $0.2 \%$ Triton X-100 in PBS, and incubated for $30 \mathrm{~min}$ at room temperature with the secondary antibody (Supplementary Table 2; 1:500 diluted in DAKO Antibody Diluent). Cells were washed with $0.2 \%$ Triton $\mathrm{X}-100$ in PBS, mounted with Prolong Gold (cat. P-36931, ThermoFisher Scientific), and visualised using a Zeiss Axioimager microscope. Stainings were replicated three times.

\section{Flow cytometry}

Day 12 PSC progeny were detached with accutase for $35 \mathrm{~s}$ at $37^{\circ} \mathrm{C}$. Accutase was inactivated by incubating cells in LDM $+10 \%$ FBS for 6 min at room temperature. Cells were washed with PBS and resuspended in PBS with $1 \%$ BSA (cat. A7979-50 ml, Sigma Aldrich). Cells were incubated with primary antibodies (Supplementary Table 2) for $30 \mathrm{~min}$ at $4{ }^{\circ} \mathrm{C}$, washed with PBS with $1 \%$ BSA and incubated with secondary antibodies (Supplementary Table 2) for $30 \mathrm{~min}$ at $4{ }^{\circ} \mathrm{C}$. Viability was assessed by propidium iodide (cat. 81845-25MG, Sigma Aldrich). Flow cytometry was performed using a BD FACSCanto ${ }^{\text {TM }}$ II HighThroughput Sampler. Cells were gated according to the strategy depicted in Supplementary Fig. 3. Two-sided paired Student's $T$ tests were applied on population median intensities (replicates: $N=15$ for CD32B and $N=14$ for MRC1).

\section{Tube formation assay}

Wells of 24-well plates were coated with undiluted Matrigel ${ }^{\circledR}$ hESC-Qualified Matrix (protein concentration approximates $10 \mathrm{mg} / \mathrm{ml}$; cat. 354277, Corning®). Matrigel was solidified by incubation for at least $10 \mathrm{~min}$ at $37^{\circ} \mathrm{C}$. Day 12-ECs (H9-ETV2 and H9-ETV2-SPI1) were seeded on the matrigel at a density of 150,000 cells per well. After $20 \mathrm{~h}$, cells were fixed with $4 \%$ paraformaldehyde, washed with PBS, and permeabilised with $0.2 \%$ Triton X-100 in PBS for 30 min. Cells were washed with PBS and blocked with $0.1 \mathrm{M}$ glycine in PBS for $30 \mathrm{~min}$. Subsequently, cells were incubated with $2 \mathrm{U} / \mathrm{ml}$ phalloidin (Supplementary Table 2), $2.5 \mu \mathrm{g} / \mathrm{ml}$ DAPI (Supplementary Table 2), and $1 \%$ BSA in PBS for $90 \mathrm{~min}$ in the dark at room temperature. Cells were washed with PBS and tubes visualised by confocal imaging with a Zeiss LSM 880-Airyscan.

\section{Scanning electron microscopy}

ETV2-ECs and ETV2-SPI1-ECs were cultured on coverslips until day 12 of differentiation with VEGFA (50 ng/ $\mathrm{ml}$; cat. 100-20, Peprotech). BALB/c LSECs were cultured for $1.5-2 \mathrm{~h}$ after isolation, with $(40 \mathrm{ng} / \mathrm{ml}$, cat. V451210UG, Sigma Aldrich). Cells were washed thoroughly with PBS to avoid protein and serum remnants. Next, cells were immersed in $500 \mu \mathrm{l}$ PBS and fixed for $10 \mathrm{~min}$ with $500 \mu \mathrm{l}$ of $5 \%$ glutaraldehyde in $66 \mathrm{mM}$ cacodylate buffer. The fixative solution was removed and cells maintained overnight at $4{ }^{\circ} \mathrm{C}$ in $2.5 \%$ glutaraldehyde in $66 \mathrm{mM}$ cacodylate buffer. Cells were washed with $0.1 \mathrm{M}$ cacodylate buffer at room temperature and post-fixed for $2 \mathrm{~h}$ at room temperature with $1 \% \mathrm{OsO}_{4}+1.5 \% \quad \mathrm{~K}_{4} \mathrm{Fe}(\mathrm{CN})_{6}$ in $0.1 \mathrm{M}$ cacodylate buffer. After post-fixation cells were washed with $0.1 \mathrm{M}$ cacodylate buffer and dehydrated with increasing ethanol concentrations (30-50-70-90-100-100\%) with $5 \mathrm{~min}$ incubation per concentration. Finally, the samples were critical point dried in a Leica CPD300 critical point dryer and the coverslips with cells mounted on pin stubs with carbon stickers. Cells were observed and imaged in a Zeiss Sigma scanning electron microscope at an accelerating voltage of $5 \mathrm{kV}$. Pore diameters were counted and measured using ImageJ and the EBImage R package.

\section{FSA-FITC uptake assay}

ETV2-ECs and ETV2-SPI1-ECs were cultured until day 12 of differentiation on Matrigel-coated wells in a 24-well plate. BALB/c LSECs were isolated and plated as well on collagen at 200,000 cells per well in a 24-well plate in $0 \%$ FBS-DMEM. After $1 \mathrm{~h}$ of LSEC plating, ETV2-ECs, ETV2-SPI1-ECs, and LSECs were supplemented with $10 \mu \mathrm{g} / \mathrm{ml} \mathrm{FSA-FITC} \mathrm{(prepared} \mathrm{as} \mathrm{described} \mathrm{in} \mathrm{ref.}{ }^{47}$ ) and 
1:1000 IncuCyte ${ }^{\circledR}$ NucLight Rapid Red Reagent (cat. 4717, Sartorius). Cells were imaged hourly over a period of $24 \mathrm{~h}$ using the IncuCyte Cell analysis system (Essen Bioscience). Quantification was performed with the IncuCyte Cell analysis software. This experiment was repeated twice. Statistical differences were assessed by mixed ANOVA.

\section{IgG-AF555 uptake assay}

H9-ETV2 and H9-ETV2-SPI1 PSCs were differentiated to ECs as described above. On day 12 of differentiation cells were washed with PBS and incubated for $2 \mathrm{~h}$ at $37^{\circ} \mathrm{C}$ with $100 \mu \mathrm{g} / \mathrm{ml} \mathrm{IgG} \mathrm{(conjugated} \mathrm{with} \mathrm{Alexa} \mathrm{Fluor} \mathrm{555;}$ Supplementary Table 2) in $\mathrm{LDM}+2 \% \mathrm{FBS}+5 \mu \mathrm{g} / \mathrm{ml}$ doxycycline. Cells were washed with PBS and fixed with 4\% paraformaldehyde. For imaging intended for statistical assessment of uptake, samples were additionally stained with DAPI. CellProfiler version 3.1.9 ${ }^{48}$ was used for segmentation of the red channels using the Watershed algorithm. Mean intensities were calculated per object. For confocal imaging, samples were additionally stained with DAPI, phalloidin, and an anti-RAB5 antibody to detect endosomes (Details in Supplementary Table 2). This experiment was repeated twice. Co-localisation of RAB5 and IgG-AF555 was quantified using the JaCoP ImageJ plug-in, as described ${ }^{49}$. The Pearson's correlation coefficient was computed after thresholding.

\section{Statistical analysis of RT-qPCR}

For all pair-wise comparisons (e.g., in the FLI1 and ERG overexpression and VEGFA addition experiments), we applied a Student's $T$ test. Statistical significance of the SPI1 lentiviral titre gradient on FCGR2B and LYVE1 expression was assessed using linear modelling. Timecourse comparisons of RT-qPCR data and FITC-FSA were assessed by mixed ANOVA. Sample sizes were a priori decided through power analysis simulations in R, given a minimal effect size of interest of two cycle thresholds, an average standard deviation of one cycle threshold, a significance level of 0.05 , and a minimally required power of 0.8 .

\section{Results}

\section{ETV2 overexpression fates pluripotent stem cells to the endothelial lineage}

To effectively differentiate PSCs into ECs, we recombined a doxycycline-inducible ETV2 overexpression cassette in H9 ESC already containing an FRT-flanked exchange cassette (Fig. 1A), as described by Ordovas et al. $^{41}$. Resulting ECs are further referred to as ETV2ECs. We assessed the endothelial differentiation (Fig. 1B) by RT-qPCR (Fig. 1C). ETV2 was more than a 1000-fold overexpressed from day 2 of differentiation. As expected, expression levels of the pluripotency markers NANOG and POU $5 F 1$ decreased by $>90 \%$ within 4 days of differentiation (Supplementary Fig. 4A). On day 6 of differentiation expression levels of the endothelial markers, i.e., PECAM1, CDH5, and VEGFR2, and endothelial TFs, i.e., FLI1 and ERG, were induced and remained stable (Fig. $1 C)$. We confirmed expression of CD31 by immunostaining (Supplementary Fig. 4B). Low to no staining was observed for the LSEC markers CD32B and MRC1. Furthermore, tube formation, a key feature of ECs, was observed after seeding ETV2-ECs on matrigel (Fig. 1D).

\section{CenTFinder workflow}

To identify and rank TFs required for fating ETV2-ECs towards LSECs, we designed a computational strategy (Fig. 2) to perform a meta-analysis on publicly available or inhouse transcriptional data from various sources of ECs and LSECs (Supplementary Table 3). To ensure that the identified TFs play a 'master regulator' role in fating ECs to LSECs, we combined various computational methods, such as gene co-expression, TF binding motif enrichment, as well as differential expression analyses. Aside from TF rankings for LSECs, we also constructed TF rankings (Supplementary Table 4) for all other EC types that were included in the meta-analysis (Supplementary Table 3). The entire workflow is available as the CenTFinder R package on Github (https://github.com/jonathandesmedt92/CenTFinder).

\section{Identification of LSEC markers by combination of microarray and single-cell RNAseq analyses}

We downloaded, filtered, and normalised relevant microarray data (Fig. 2. left panel and Fig. 3A, and metadata in Supplementary Table 3). Next, we represented the gene expression profiles of all arrays $(N=279)$ by $t$-distributed Stochastic Neighbour Embedding ( $t$ $\mathrm{SNE}$ ), which shows great heterogeneity and sets the human primary LSECs apart from all other EC subtypes (Fig. 3B). Differential expression analysis between LSECs and all other EC types (Fig. 3C, D) confirmed established, as well as only recently identified LSEC marker genes, such as CLEC4G, FCGR2B, STAB2, MRC1, CLEC4M, F8, LYVE1, FCN2, FCN3, OIT3, CLEC1B, DNASE1L3, and $G P R 182^{45,50,51}$. In addition, we could identify putative novel LSEC marker candidates of which we validated LSEC-specific expression in two recently published liver scRNAseq datasets ${ }^{42,45}$ (Fig. 3E). Several putative markers were only expressed in the LSEC population, i.e., $\mathrm{CDH} 5+$ $K D R+C L E C 4 G+$ cells $^{42,51}$. In addition, some candidate markers, such as $A L B$ and $A P O A 2$, were expressed in hepatocytes rather than LSECs. By merging fold changes of the CenTFinder and scRNAseq datasets, we generated a consensus list of scRNAseq-validated LSEC markers that were at least sevenfold higher expressed in LSECs compared to the other cells in the respective datasets ${ }^{42,45}$ (Fig. 3F). 
A

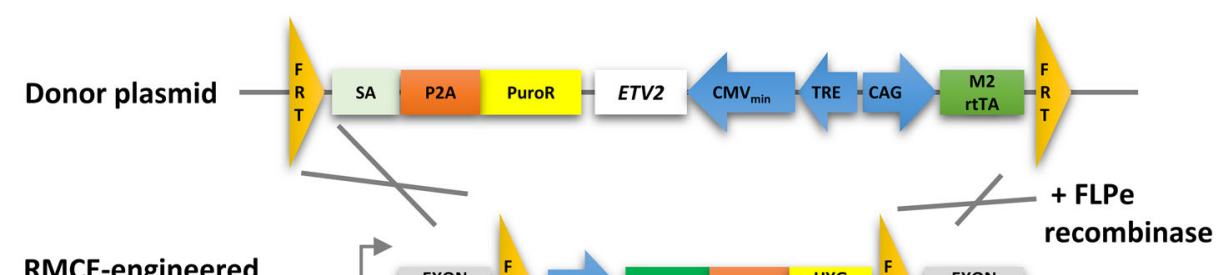
AAVS1 locus in $\mathrm{H9}$

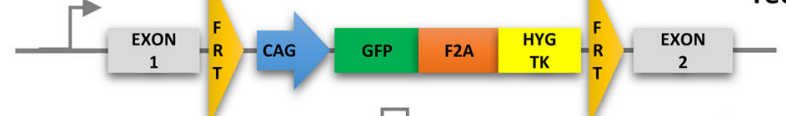

Puromycin and FIAU selection

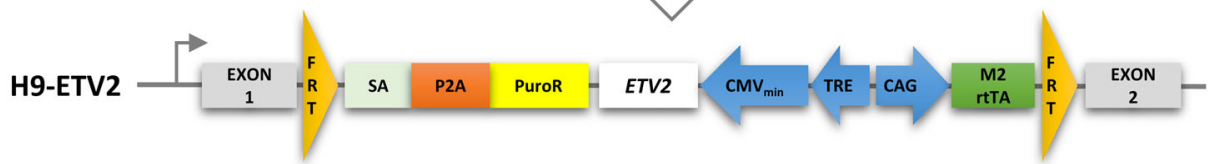

B

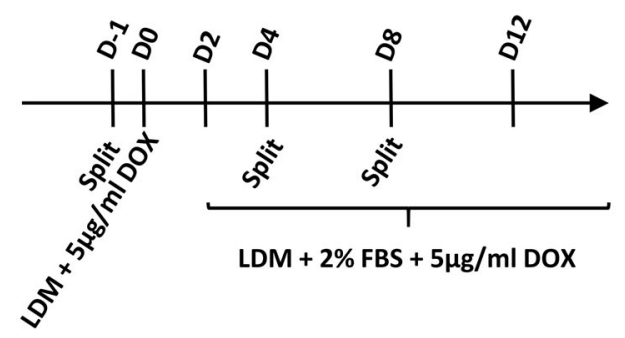

D

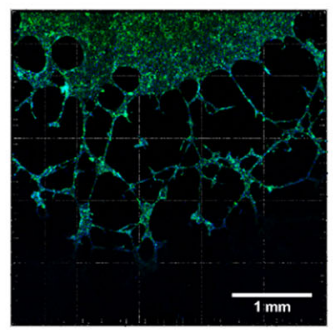

C

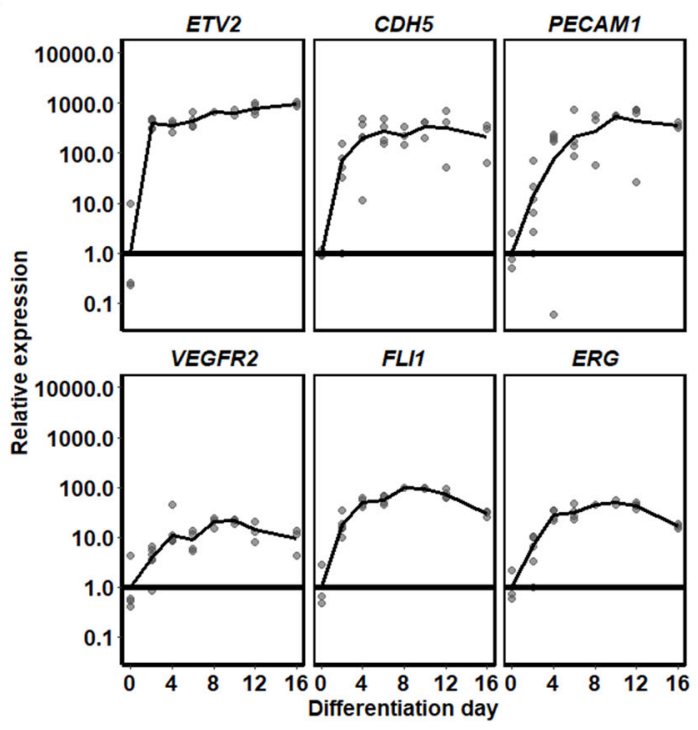

Fig. 1 Generation and characterisation of ETV2-ECs. A H9 human embryonic stem cells engineered with an RMCE cassette were recombined with a doxycycline-inducible ETV2 overexpression cassette (method described in and figure adapted from Ordovas et al. ${ }^{41}$ ). (FRT Flippase recognition target, SA splice acceptor, PuroR puromycin resistance gene, TRE tetracycline response element, M2rtTA M2 reverse tetracycline transactivator.) B Schematic representation for PSC differentiation into endothelial cells. C Gene expression (RT-qPCR) profile of ETV2, the endothelial markers CDH5, PECAM1, and VEGFR2, and the endothelial TFS FLI1 and ERG. Samples were collected every 2-4 days of differentiation until day 16 with $N=3$ biological replicates per time point. Expression is relative to that of day 0 of differentiation (i.e., stem cells). D Confocal image of phalloidin-stained ETV2-ECs in Matrigel showing tube formation.

WGCNA and RcisTarget analysis identified SPI1 and IRFs as central to immune response transcriptional modules specific for LSECs

TFs were ranked by fold change (LSECs compared to other ECs in the microarray meta-analysis (Fig. 3D). However, such ranking identifies both putative TFs required for fating ECs to LSECs, as well as broadly acting TFs (e.g., JUN and FOS). Furthermore, transcripts of cell types other than LSECs may also bias this ranking as assessed by analysis of published single-cell RNAseq data ${ }^{42}$ (Supplementary Fig. 5). We subsequently used a gene regulatory network (GRN) approach (WGCNA) to select more specific TFs that might guide LSEC differentiation.
WGCNA is a tool that clusters genes with correlated expression patterns into modules that are, as a result, highly enriched for specific GO terms and pathways. We subjected the expression matrix to $\mathrm{WGCNA}^{38}$, to identify transcriptional modules, their associated GO terms and central TFs (i.e., TFs with a high module eigengene). We identified seven transcriptional modules (Fig. 4A) that were each active in at least one of the EC types included in the meta-analysis (Fig. 4B; excluding the grey module containing unassigned genes). By GO analysis, three of the modules were related to immune response genes (i.e., red, green, and brown), two to cell cycle genes (i.e., (S-G2-M) yellow and (G1-S transition) black), one to RNA 


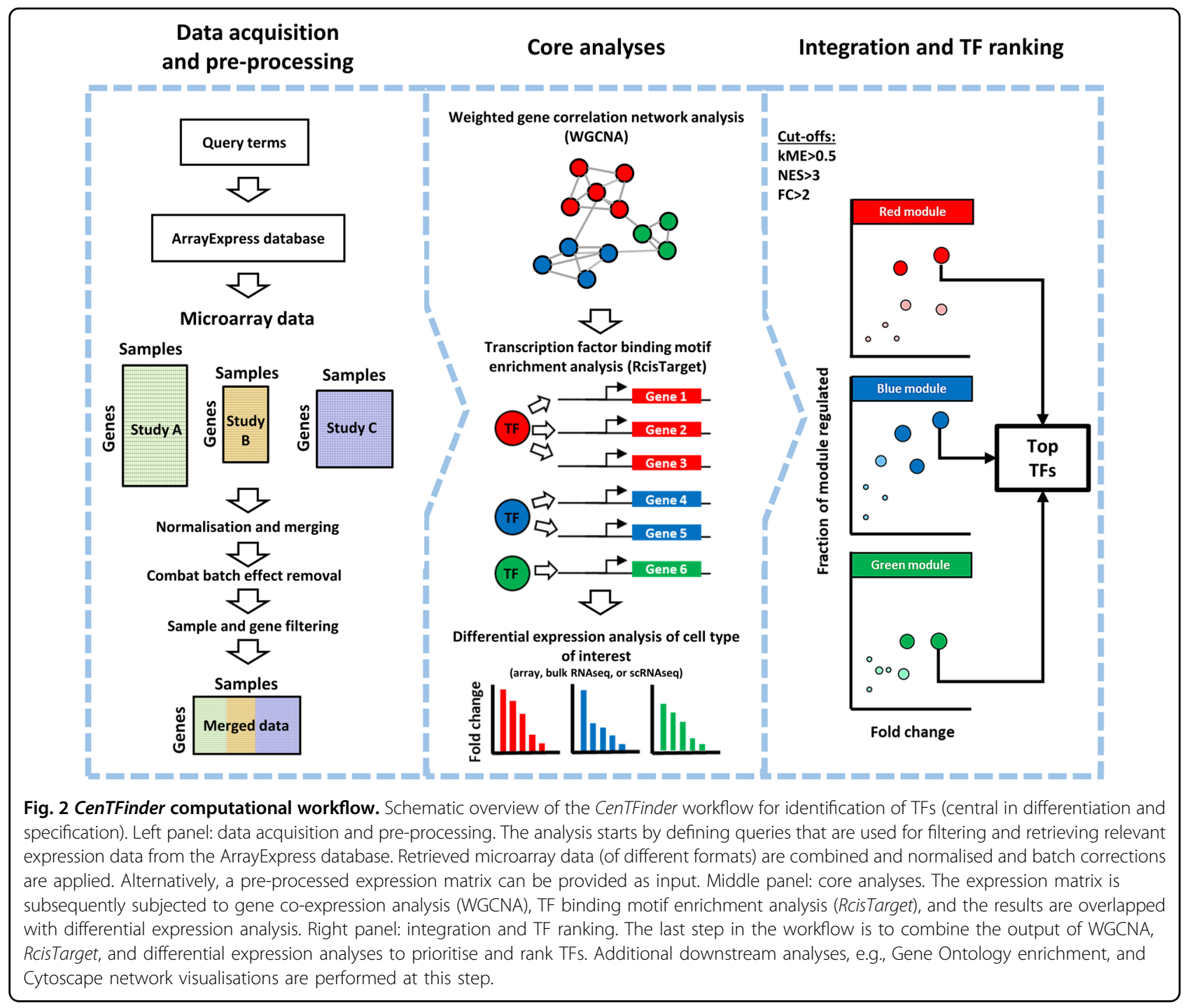

metabolism and translation (i.e., blue), and one to development, gene expression regulation, and focal adhesions (i.e., turquoise; Supplementary Fig. 6). Comparison of module activities (calculated with gene set variation analysis, GSVA $)^{52}$ demonstrated that the modules related to immune response, development, and focal adhesions were more active in LSECs compared to other ECs. In contrast, the (S-G2-M) cell cycle and the RNA metabolism modules were less active in LSECS (Fig. 4B, C). Interestingly, the immune clusters contained several C-type lectin receptors, such as CLEC1OA, CLEC2D, CLEC4A, CLEC4E, CLEC5A, and CLEC7A, as well as several immunoglobulin receptors, such as FCER1G, FCGR1A, FCGR2A, FCGR2B, and FCGR3B (immune clusters represented in Fig. 4D).

To avoid interference of spurious correlations due to technical or biological noise, we applied RcisTarget ${ }^{39}$ to each of the WGCNA modules (Fig. 4E). RcisTarget identifies TFs for which there is an enriched binding motif presence in the cis-regulatory regions of the genes in a given gene set. Only TFs with a network enrichment score (NES) > 3 were considered and TFs that regulate higher fractions of their respective modules were deemed more important or influential. In the immune response modules related to cytokine signalling and secretion, SPI1 had binding motifs in most of the genes in each of the respective clusters. In the immune response module related to inflammasomes, most genes were regulated by IRF TFs.

To further narrow down more promising TFs, we used bulk RNA-sequencing data to identify which of these TFs are differentially expressed between ETV2-ECs and LSECs (Fig. 5A, B). Next, we made an overlap between the cluster central and RcisTarget-enriched TFs that were expressed more in LSECs compared to ETV2-ECs (Fig. 5C). All TFs in the development and focal adhesions module were less 
A

B
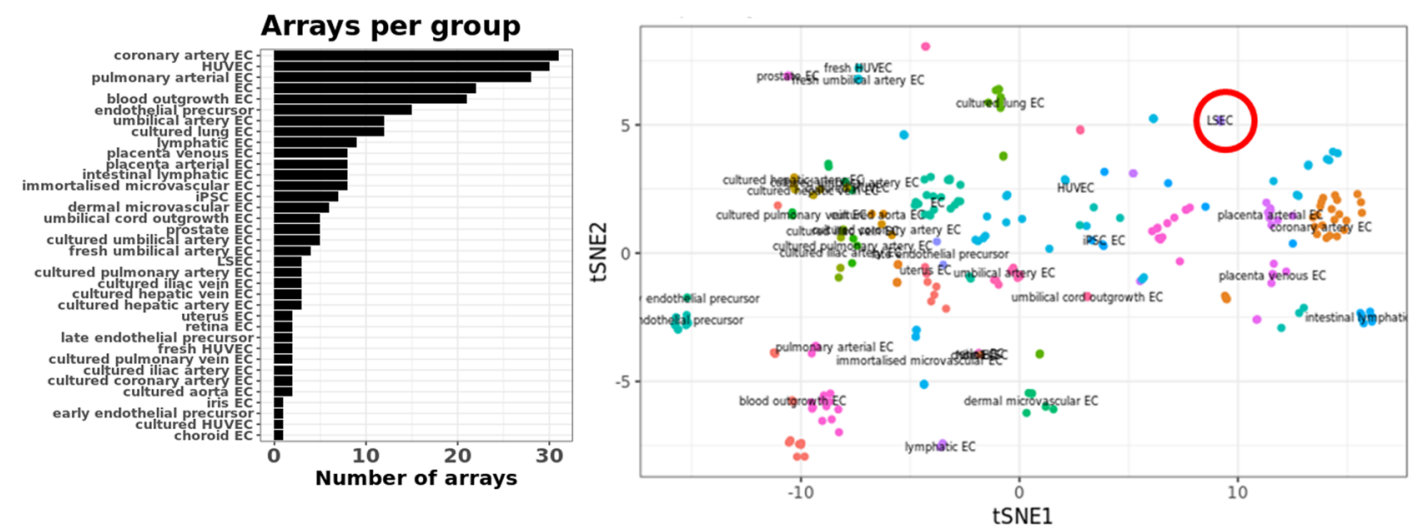

C

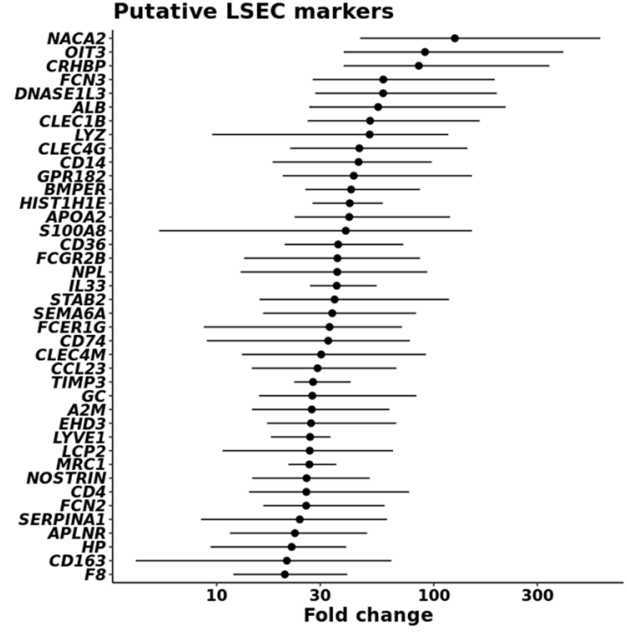

D

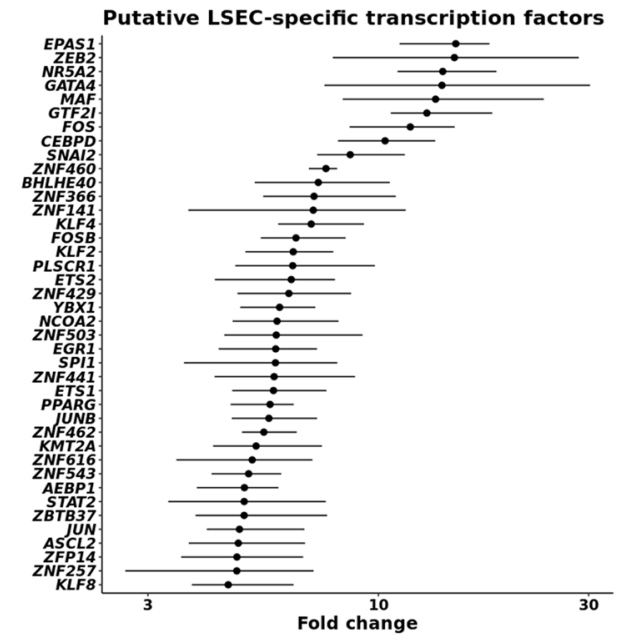

$\mathbf{F}$
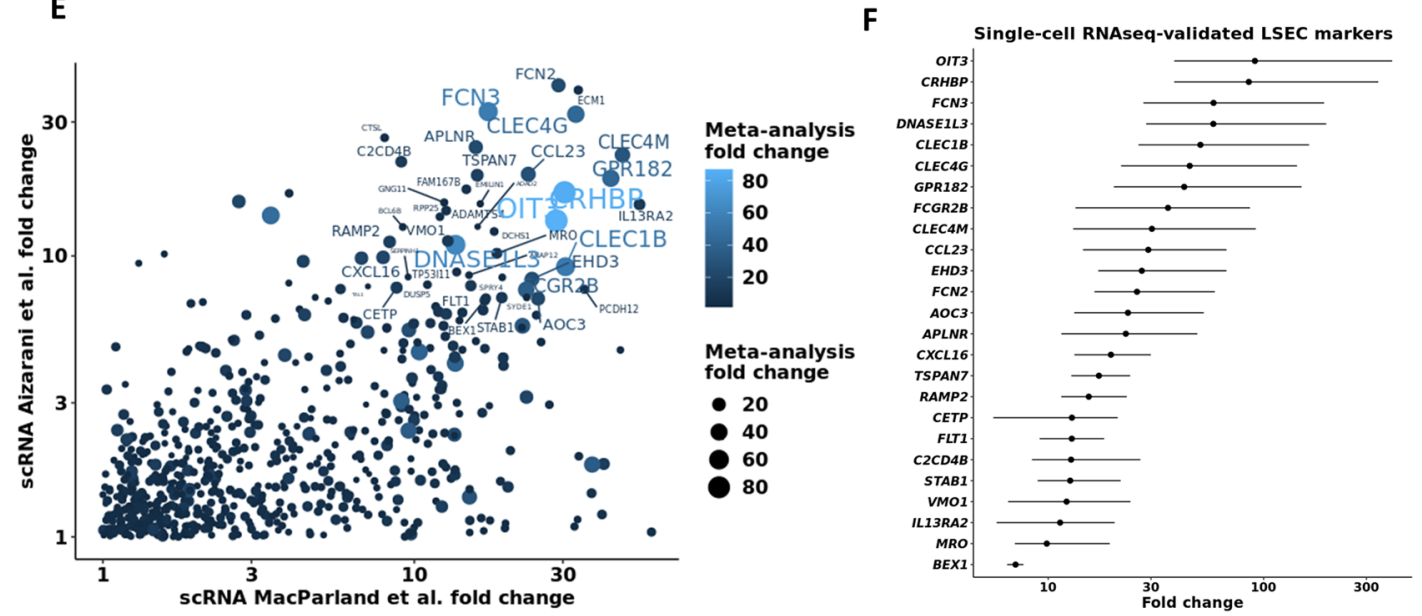

Fig. 3 Intersection between microarray meta-analysis and single-cell RNAseq identifies putative LSEC markers. A Bar chart indicating the number of microarrays per endothelial cell type that was included in the CenTFinder analysis (total number of arrays $=279$ ). B $t$-SNE representation of all endothelial cells used in the analysis (LSECs circled in red). LSEC microarray data were contrasted to the expression data of all other endothelial cells used in the meta-analysis. Line charts show putative markers (C) and TFs (D) with increased expression in LSECs compared to all other endothelial cells (assessed by Wilcoxon rank-sum test). Putative markers and TFs were sorted by fold change. Dots indicate the mean fold change between LSECS and all other ECs. Lines span the interquartile range. E Overlap of genes that are specifically expressed in LSECS in the single-cell RNA-sequencing studies and in the microarray meta-analysis. The identified genes are more expressed in LSECs compared to all other endothelial cells, as well as compared to all other parenchymal and non-parenchymal liver cells. X-and $Y$-axes denote the two single-cell RNAseq studies ${ }^{42,45}$. F LSEC marker ranking by fold change compared to all other ECs in the microarray meta-analysis. For this analysis, genes were ranked only if in each of the studies they were more than sevenfold higher expressed in LSECS compared to the other cells. Dots indicate the mean fold change between LSECs and all other ECs. Lines span the interquartile range. 


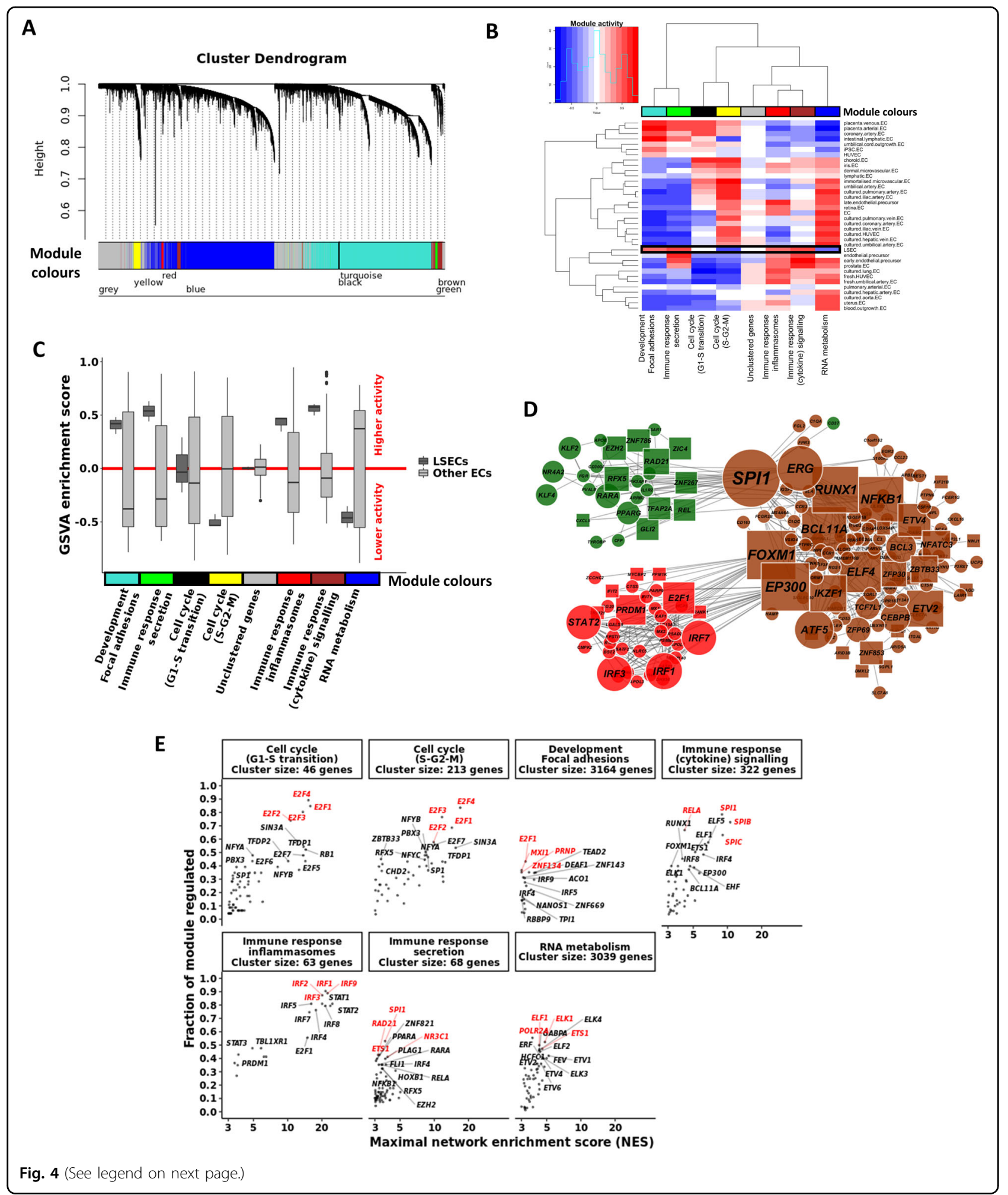

expressed in LSECs compared to ETV2-ECs. In the immune reponse (cytokine signalling and secretion) modules SPI1 was the most highly differentially expressed (469-fold, $p<0.001$; cluster centrality $k \mathrm{ME}=0.6$ and $k \mathrm{ME}=0.9$, respectively, and $\mathrm{NES}=8.97$ and $\mathrm{NES}=3.69$ respectively). In the immune response (inflammasomes) module, IRF1, IRF7, and STAT2 passed the filtering criteria. An analysis of the number of downstream targets for 
(see figure on previous page)

Fig. 4 Gene co-expression (WGCNA) and transcription factor binding motif (RcisTarget) analyses. A WGCNA cluster dendrogram visualising the gene co-expression modules identified in the microarray meta-analysis. These modules are active in at least one of the endothelial cell types. The grey module contains genes unassigned to any module due to low correlative values. B Heatmap and clustering of the gene set variation analysis (GSVA) activity scores for each of the modules and for each of the cell types. Module colours correspond with those from the cluster dendrogram in A. Black box identifies module activities in LSECS. C Boxplots of the GSVA enrichment scores for each of the modules, comparing LSECs to all other endothelial cell types. D Cytoscape visualisation of the LSEC-specific immune response modules. Edges indicate a regulatory link identified by RcisTarget. TF node size relates to the number of downstream targets. For the sake of simplicity, only nodes of the 20 most differentially expressed downstream targets are shown for each TF. Nodes were coloured according to their module colour. TFs belonging to other modules but with a kME $>0.5$ for either the brown, red, or green modules were included as well and coloured accordingly. Circular (square) nodes represent genes that are higher (lower) expressed in LSECs compared to ETV2-ECs. E Enrichment of TF binding motifs was calculated for each of the WGCNA gene coexpression modules. Each dot represents an enriched TF binding motif with the $x$-axis indicating the RcisTarget network enrichment score (NES) and the $y$-axis indicating the fraction of genes in the respective module that has binding motifs for the respective gene. For genes with multiple enriched motifs, only the motif with the highest NES is shown. Top TFs are highlighted in red.

A
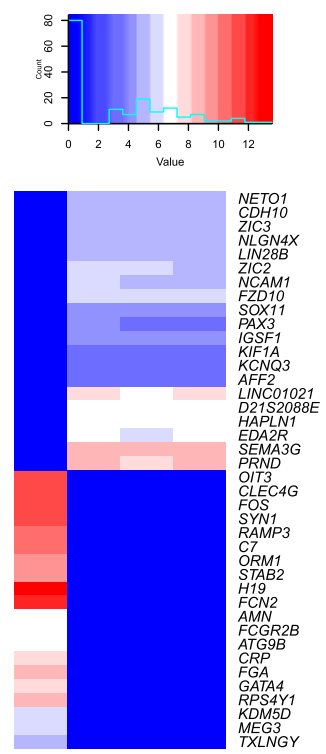

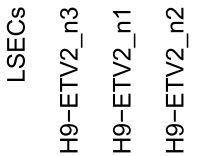

B

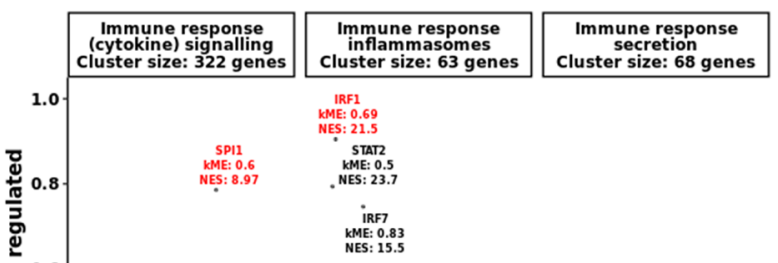

C
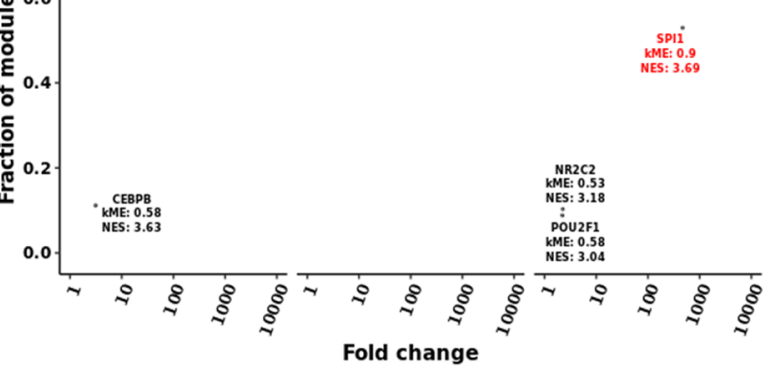

Fold change
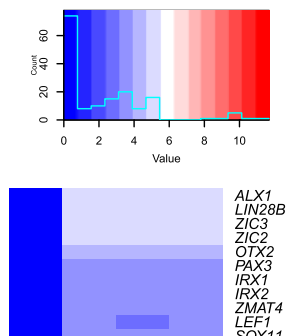

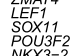

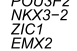

ZIC1

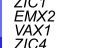

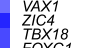

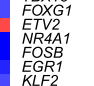
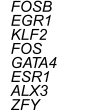

ALX
ALY
LYY
HNG
ZNE5

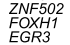

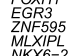

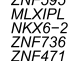

ZNF 471
PRO2
ZNF667

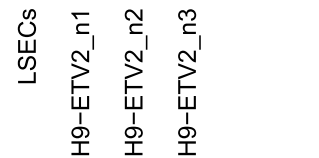

Fig. 5 CenTFinder analysis identifies SPI1 and IRF TFs as major LSEC regulators. A Heatmap of the top 20 most upregulated and downregulated genes in LSECs compared to ETV2-ECS. B Heatmap of the top 20 most upregulated and downregulated TFs in LSECs compared to ETV2-ECs. C CenTFinder core analysis results were combined. Each dot represents a TF that was differentially and higher expressed in LSECS compared to ETV2ECs. In addition, only TFs with $k M E>0.5$ and NES $>3$ were considered. SPI1 was identified as the central TF in the immune response clusters related to cytokine signalling and secretion. IRF TFs were central to the immune response cluster related to inflammasomes. Top TFs are highlighted in red.

the different TFs demonstrated that PU.1 regulates considerably more genes than the other TFs (Supplementary Fig. 7). Furthermore, inspection of RcisTarget's gene-motif database (hg19-tss-centred-10kb-7-species.mc9nr.feather) showed that, of all selected TFs, only PU.1 has binding motifs for which some of the LSEC markers are highly ranking targets (Supplementary Fig. 8). This was confirmed by in silico analysis of available ChiP-Seq data (downloaded from ChiP-Atlas https://chip-atlas.org/; Supplementary Fig. 9).

In conclusion, the microarray EC and LSEC metaanalysis identified SPI1 and IRFs to be candidate master regulators of LSEC fating. As the comparison of LSECs and ETV2-ECs also identified SPI1 to be the highest ranking TF in LSECs, subsequent studies were done to validate SPI1 as a master regulator of LSEC fating. 


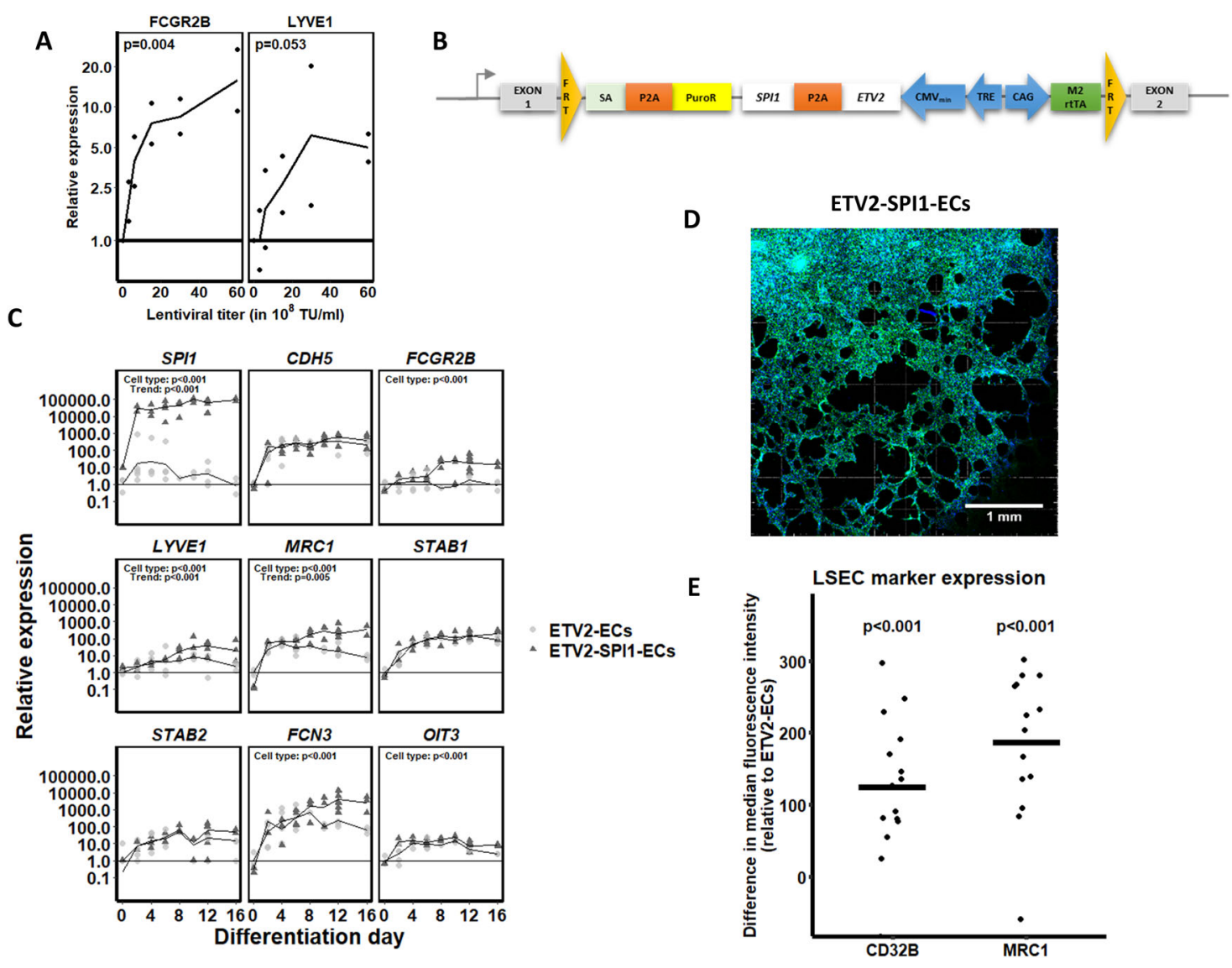

Fig. 6 Characterisation of (liver sinusoidal) endothelial cell markers in ETV2-ECs and ETV2-SPI1-ECs. A ETV2-ECs were transduced on day 6 of differentiation with increasing titres of the SPI1-encoding lentiviral vector. The effect of SPI1 overexpression on the LSEC markers FCGR2B and LYVE1 is shown. Statistical analysis by linear modelling (expression titre); $N=2$ biological replicates per concentration. B ETV2-SPI1 construct engineered into the AAVS1 safe harbour locus. Recombination was performed analogously to Fig. 1A. (FRT Flippase recognition target, SA splice acceptor, PuroR puromycin resistance gene, TRE tetracycline response element, M2rtTA M2 reverse tetracycline transactivator). C Time-course comparison between ETV2-ECS and ETV2-SPI1-ECS for the overexpressed SPI1, for the endothelial cell-specific gene CDH5, for the known LSEC markers FCGR2B, LYVE1, MRC1, STAB1, and STAB2, and for novel LSEC markers, such as FCN3, and OIT3. Statistical significances were assessed by mixed ANOVA. Undetected mRNA values were represented with a relative expression of 1. The $x$-axes denote the days of differentiation. The differentiation protocols for ETV2-ECs and ETV2-SPI1-ECS are identical. D Tube formation assay on day 12 of differentiation of ETV2-SPI1-ECs. E LSEC marker expression (CD32B and MRC1) in ETV2-SPI1-ECs compared to ETV2-ECs. Chart indicates the median fluorescence intensity shift for CD32B and MRC1 of ETV2-ECs and ETV2-SPI1-ECs on day 12 of differentiation (replicates: $N=15$ for $C D 32 B$ and $N=14$ for MRC1). The CD32 and MRC1 positive cells were gated from single-cell, PInegative, and VE-cadherin-positive populations (gating strategy in Supplementary Fig. 3).

\section{SPI1 overexpression induces LSEC marker expression}

To evaluate if SPI1 can drive LSEC specification and maturation in ETV2-ECs, we cloned the SPI1 coding sequence into a pLVX backbone and transduced ETV2ECs on day 6 of differentiation with the resulting lentiviral vector. Clear upregulation of the LSEC markers FCGR2B and LYVE1 was observed (Fig. 6A). To avoid the toxicity associated with lentiviral transduction, and to ensure that all cells contained the SPI1 TF, we next recombined a doxycycline-inducible cassette for both ETV2 and SPI1 in the AAVS1 safe harbour locus, to generate ETV2-SPIECs (Fig. 6B). Combined induction of ETV2 and SPI1 did not impact general EC differentiation (Supplementary Fig. 10). Specifically, expression of NANOG and POU5F1 decreased by $>90 \%$ on day 4 (Supplementary Fig. 10A) and no differences in expression of the EC markers PECAM1 and CDH5, and the EC TFs ERG, ETV2, and FLI1, were observed between ETV2- and ETV2-SPI1-ECs (Supplementary Fig. 10B). Consistently, immunostaining for CD31 was similar in ETV2 and ETV2-SPI-ECs (Supplementary Fig. 11). Transcript levels for SPI1 were $>1000$-fold higher expressed in ETV2-SPI1-ECs compared to ETV2-ECs $(p<$ 0.001; Fig. 6C). In addition, tube formation by ETV2-ECs and ETV2-SPI1-ECs was similar (Fig. 6D).

Next, we compared the expression over time of the known and the proposed novel LSEC markers (as described in Fig. 3F). RT-qPCR demonstrated that the LSEC markers FCGR2B $(p<0.001)$, LYVE1 $(p<0.001)$, 
$M R C 1(p=0.002), C R H B P(p=0.025), F C N 3(p=0.011)$, and OIT3 $(p=0.018)$ were significantly higher expressed in ETV2-SPI1-ECs compared to ETV2-ECs (Fig. 6C and Supplementary Fig. 10C). This was further substantiated by immunostaining (Supplementary Fig. 11) and flow cytometry analysis (Fig. 6E and Supplementary Fig. 3), which showed a higher expression of CD32B and MRC1 in the ETV2-SPI1-ECs compared to the ETV2-ECs. CLEC4G, a highly specific LSEC marker, was however not expressed in either ETV2-ECs or ETV2-SPI1-ECs (data not shown).

As ETV2 is only expressed until E10.5 in the mouse ${ }^{53}$, we tested if persistent induction of ETV2 might prohibit differentiation of ETV2-ECs into ECs with a more mature, LSEC-like phenotype. Therefore, to replace ETV2 overexpression with TFs that are expressed in adult ECs, we cotransduced lentiviral vectors encoding the SPI1, FLI1, and/or ERG genes in ETV2-ECs on day 6 of differentiation, followed by doxycycline omission, and hence loss of ETV2 expression, as of day 8. Loss of ETV2 expression caused a significant drop $(>90 \%, p<0.01)$ in PECAM1 expression unless cells were cotransduced with FLI1 and/ or ERG (Supplementary Fig. 2A). Combined induction of $E T V 2, E R G$, and SPI1 led to the increased expression of $M R C 1$ and OIT3 $(p<0.05)$. In contrast, combined induction of FLI1 and SPI1 decreased expression of FCN3 and DNASE1L3 ( $p<0.05$; Supplementary Fig. 2B, C). Overall, replacement of ETV2 overexpression with FLI1 or ERG overexpression could maintain the endothelial phenotype, but did not further enhance LSEC marker expression.

As the differentiation assay did not include VEGFA, and VEGFA may be beneficial for the LSEC phenotype ${ }^{17,54}$, we repeated differentiations with inclusion of VEGFA from day 6 onwards. Expression of LYVE1 in ETV2-SPI1-ECs was significantly induced by VEGFA (19.71-fold; $p=$ 0.005; Supplementary Fig. 1). However, VEGFA did not significantly induce other LSEC markers, such as FCGR2B, STAB1, and STAB2.

\section{Doxycycline-inducible SPI1 overexpression improves LSEC features in ETV2-ECs}

To assess LSEC functionality of ETV2-SPI1-ECs, we performed assays for the uptake of formaldehyde-treated FITC-coupled albumin (FSA-FITC; Fig. 7A-C), as well as IgG-AF555 (Fig. 7D-F). FSA-FITC uptake, which is scavenger receptor-mediated, is efficient in primary mouse LSEC (Fig. 7B). In PSC-derived ETV2-ECs, FSA-FITC uptake was enhanced in ETV2-SPI1-ECs when compared with ETV2-ECs $(p<0.001)$. In addition, when VEGFA was supplemented to the culture media, the uptake of FSAFITC was increased in both cell populations $(p<0.001$; Fig. 7C). Furthermore, CD32B-mediated IgG uptake was observed in only $22.21 \%$ (301/1355) of ETV2-ECs (Fig. $7 \mathrm{D}, \mathrm{E})$, while we could detect IgG-AF555 uptake in
$68.60 \%(933 / 1360)$ of ETV2-SPI1-ECs $(p=0.022)$, with the IgG-AF555 fluorochrome co-localised with endosomes identified by a RAB5 antibody (Pearson correlation for co-localisation: $0.733 \pm 0.055$; Fig. $7 \mathrm{G}$ ). In addition, the IgG-AF555-positive ETV2-SPI1-ECs were significantly brighter than the IgG-AF555-positive ETV2-ECs $(p<$ 0.001; Fig. 7F).

We also performed scanning electron microscopy on ETV2-ECs and ETV2-SPI1-ECs (with mouse LSECs as control) to determine if induction of SPI1 induced fenestrae formation (Fig. 7H). Mouse LSECs showed clear fenestrations grouped in sieve plates. LSEC fenestration diameter was $122.73 \pm 48.26 \mathrm{~nm}$ (Fig. 7I). However, neither ETV2- or ETV2-SPI1-ECs displayed fenestrations. Some transcytoplasmic holes were observed in ETV2-ECs and ETV2-SPI1-ECs, but these holes were much smaller, i.e., $28.38 \pm 18.02$ and $33.55 \pm 22.79 \mathrm{~nm}$, respectively, and likely due to SEM preparation artefacts, as previously indicated by Elvevold et al. ${ }^{55}$. We did note a clear morphological difference; ETV2-SPI1-ECs appeared more rounded with a few large cellular protrusions radiating from the main cell body, while ETV2-ECs appeared as flat cells with no or small and thin protrusions.

\section{Discussion}

To identify TFs that are crucial for LSEC fating, we created a computational workflow (CenTFinder) that applies several bioinformatic tools on (a meta-analysis of) transcriptomics datasets. Our analysis identified SPI1 as a key $\mathrm{TF}$ in a number of immune response pathways characteristic for LSECs. We demonstrated that forced expression of SPI1 in PSC-derived ECs can partially fate PSC-ECs to an LSEC-like phenotype.

Classically, in differential expression analyses genes or TFs are ranked by fold change and/or $p$ value. However, such ranking may not necessarily yield (all) relevant TFs, as is exemplified by SPI1, which did not rank in the top 20 of differentially expressed TFs in the CenTFinder analysis. TFs might be differentially expressed due to differences between in vivo and in vitro environments, or due to impurities in primary cell populations. In addition, the fold change of a given TF does not provide knowledge regarding its number of dowstream targets, regarding its relevance to the cell-of-interest's phenotype, or regarding the presence and complexity of its interactions with other proteins or signalling molecules.

To address these issues and to identify TFs that are crucial for LSEC fating, CenTFinder combines gene coexpression analysis (WGCNA), TF binding motif enrichment analysis (RcisTarget), and differential gene expression analysis. Several studies have been published that combine microarray meta-analysis with gene co-expression methods $^{56,57}$; however, to date only a single study has combined this with TF binding motif enrichment as well ${ }^{58}$. 


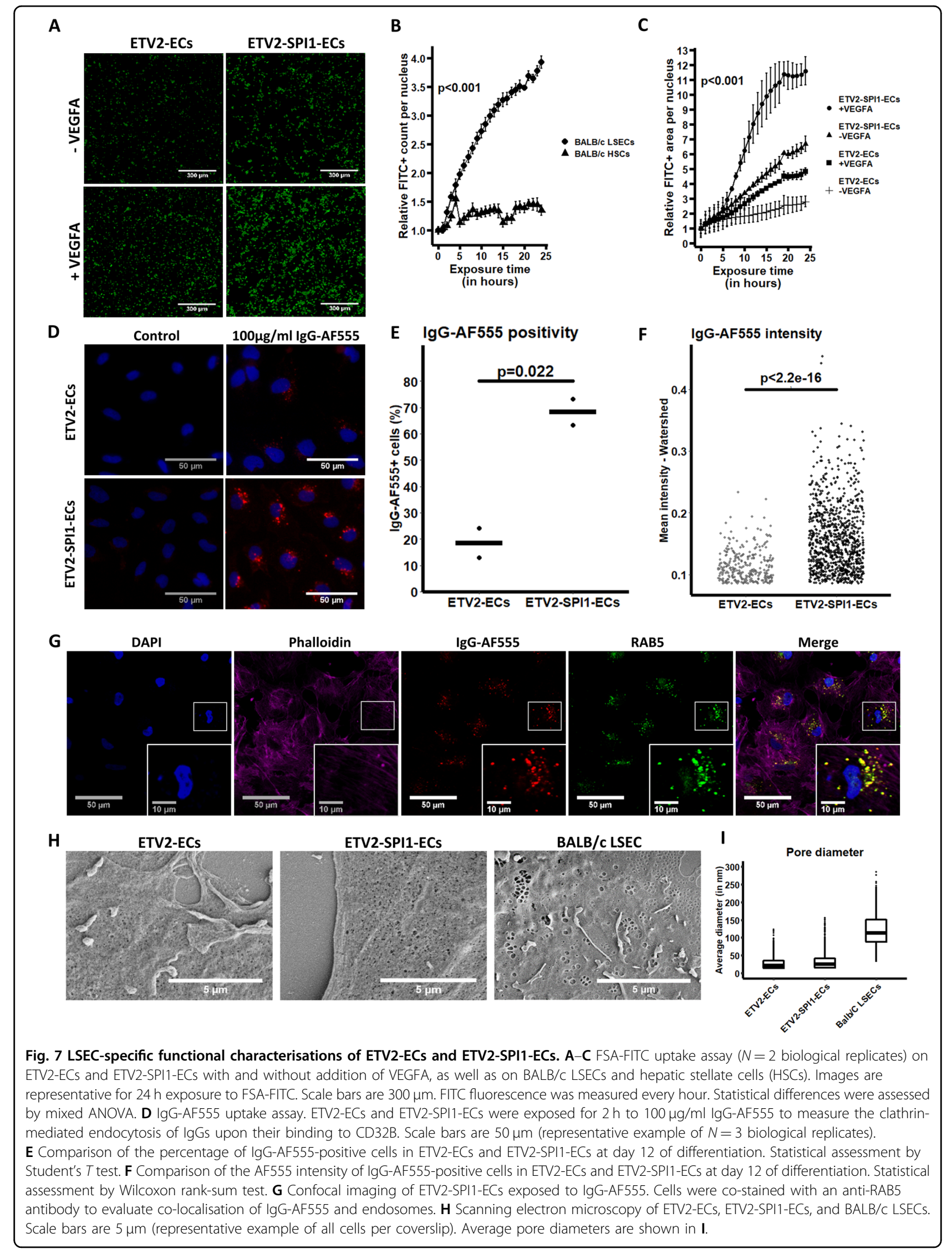


Furthermore, the SCENIC workflow ${ }^{39}$, a well-established analysis tool for single-cell RNA-sequencing data, is in essence based on similar premises.

The underlying rationale for gene co-expression analyses is that genes that are associated with the same biological process or pathway tend to be coregulated, thereby increasing the correlation between their expression patterns. Expression coregulation implies the existence of a subset of responsible upstream TFs, which hence would display higher connectivity and cluster centrality ( $k \mathrm{ME})$. Applying TF binding motif enrichment analysis on gene coexpression modules instead of the full gene set decreased the likelihood of prioritising a TF that was assigned to a module due to mere spurious correlation (e.g., correlation due to array probe position ${ }^{59}$ ). Simultaneously, it increased the likelihood of prioritising relevant TFs, as these TFs would not only be highly co-expressed with their module members, but would also have enriched binding motifs in the cis-regulatory elements of these very same module members. Furthermore, TFs with enriched binding motifs in relatively smaller clusters (such as SPI1 in our analysis) might not be detected by binding motif enrichment applied on the full list of differentially expressed genes. TFs were constrained by their centrality in a functional co-expression module and by the presence of enriched binding motifs. As TFs are assigned to a functional co-expression module, one can disregard TFs of modules that are not specifically related to the cell type of interest. For instance, in our analysis, we found that only three modules (immune response) out of seven were relevant for the LSEC phenotype, and hence we excluded TFs from other modules. Finally, we prioritised TFs that were differentially expressed between ETV2-ECs and LSECs, as we reasoned that correcting the respective module activities might suffice by mere overexpression of these TFs.

We note that such strategy could be of interest, as well in any other research aiming at improved differentiation and specification of PSC progeny towards a cell type of interest. In addition, the developed package can also identify TFs central to response to chemicals or different culturing conditions. The only limiting step in the described strategy is the number of arrays required for gene co-expression inference. As per their FAQ page (https://horvath.genetics. ucla.edu/html/CoexpressionNetwork/Rpackages/WGCNA/ faq.html), the WGCNA authors do not recommend applying WGCNA on $<20$ samples. However, to counter noise, false-positive associations, and hence incorrect TF binding site enrichments, we would use at least 100 arrays. Extensive tutorials have been made available for WGCNA and RcisTarget analysis by their respective authors. Similarly, we made a tutorial available at https:/github.com/ jonathandesmedt92/CenTFinder.

We identified seven WGCNA modules, of which six were differentially active in LSECs compared to other
ECs. Consistent with the known roles of LSECs in innate and immune functions, three modules contained genes related to immune response pathways. Interestingly, SPI1 was identified as one of the key regulators of two of these immune clusters. SPI1, the gene encoding for the PU.1 protein, is commonly believed to play a pivotal role in $\mathrm{B}$ cells, NK cells, granulocytes, dendritic cells, and macrophages $^{60-63}$. Although PU.1 and IRF TFs were recently identified as TFs that are potentially relevant for the LSEC phenotype $^{64}$, to our knowledge no group has functionally validated its role in fating ECs to LSEC-like cells.

We here provide conclusive evidence for PU.1 to be a master regulator for LSEC fating and as a key regulatory role in LSEC functionality. Over a hundred transciptional targets for PU.1 in haematopoietic progeny had already been identified by various groups ${ }^{65}$, including CLEC4G, $F C G R 2 B$, and MRC1. Although CLEC4G expression was not induced upon SPI1 overexpression in PSC-ECs, other established LSEC markers, such as FCGR2B and MRC1, were significantly induced. Furthermore, novel putative LSEC markers identified in this study (and in oth$\mathrm{ers}^{42,45,50}$ ), such as FCN2, FCN3, CRHBP, and OIT3 were also induced by PU.1. Overexpression of PU.1 induced not only increased expression of $F C G R 2 B$, but the activity of the CD32B receptor was also significantly higher, as was evidenced by IgG-AF555 uptake.

Interestingly, not all LSEC markers were induced. We noted that these marker genes were not present in the immune response modules, but in the turquoise module related to development and focal adhesions (CLEC4G, $F C N 2$, and $S T A B 1)$, or in the grey module containing unclustered transcripts (CLEC4M, CRHBP, DNASE1L3, and STAB2). In addition, of the induced LSEC markers, $F C N 3$ and OIT3 belonged to the turquoise module, and LYVE1 and MRC1 to the grey module. This could be caused by several factors. First, the Pearson correlation used for construction of the co-expression networks does not detect non-linear gene interactions. Combinatorial regulation of the LSEC markers may hence reduce the Pearson correlation of these markers with their respective regulator TFs. Expression of some of the induced and noninduced LSEC markers might thus be dependent on the interaction of PU.1, and one or more yet to be identified other TFs. Second, variables such the epigenetic status or chromatin conformation may effect correlation values as well. Third, interestingly, several LSEC markers clustered in the turquoise module, which contains genes related to substrate adhesion. Future research may address (co-) culturing in 3D cultures, and assess transcriptional changes in this module and the LSEC markers in this modules.

Finally, uptake of FSA-FITC, a commonly used functional assay for LSECs, was more evident in ETV2-SPI1-ECs, but not in ETV2-ECs, and this effect was enhanced with VEGFA supplementation, which is known to support 
in vivo LSEC differentiation ${ }^{66,67}$, as well as in vitro culture ${ }^{17}$. However, as STAB1/2 expression remained unchanged upon VEGFA addition, the mechanism could be posttranslational, for instance at the level of receptor recycling.

While PU.1 is already known to regulate expression of cytokines, antibodies, and antibody receptors in a variety of cell types, to date no group, to our knowledge, described this in LSECs. Even more, it has been described that LSECs express CD45, a common haematopoietic marker ${ }^{68}$. Considering this, the fact that PU.1 is a critical TF for haemotopoietic stem cells, and given the unique immune roles of LSECs, it raises the question whether the LSEC might descend from an intermediate cell type in the haemogenic endothelium. Future lineage tracing experiments during development will be required to help define LSEC identity and origin. Based on the very recent singlecell RNAseq data of Wang et al. ${ }^{69}$ on human developing liver, it might also be of interest to test if specific TFs that are for instance present in endocardium during development, may aid in generating LSECs from PSCs.

Further research could also address the role of other TFs that are central in the immune response modules. Moreover, TFs that appear cluster central and have enriched binding motifs within their respective modules, but did not pass stringent differential expression criteria, could also be evaluated. The activity (or mere nuclear translocation) of such TFs could be modulated by interacting small molecules or other signalling factors. Furthermore, modules with lower activity in LSECs might contain TFs that interfere with immune response modules, or TFs inhibiting the formation of fenestrae. Hence, knockdown of such TFs might further improve the LSEC phenotype.

In summary, our findings combine complementary transcriptomics analysis methods in order to maximise knowledge extraction from transcriptome datasets. For this purpose, we developed the CenTFinder $\mathrm{R}$ package which bundles meta-analysis, gene co-expression analysis, TF binding motif enrichment analysis, and differential expression analysis in one integrated pipeline, providing also a framework for identifying TFs involved in, for instance, other endothelial lineage fating of stem cells. Based on this approach, we identified SPI1 as the most highly ranked LSEC-specific TF, and demonstrated the importance of PU.1 in LSEC differentiation from PSCs.

\section{Acknowledgements}

We would like to thank Prof. Dr. Stein Aerts for his valuable input, advice, and discussions on bioinformatic strategies, Dr. Sara Aibar for her advice on the usage of RcisTarget, Dr. Cristina Øie for her critical assessment of and advice on the IgG uptake assays, and Dr. Aernout Luttun for his well-valued advice. Furthermore, we would like to acknowledge the VIB Nucleomics Core for the RNA-sequencing, related quality control, and fruitful discussions. Confocal Images were recorded on a Zeiss LSM 880-Airyscan (Cell and Tissue Imaging Cluster (CIC)), supported by Hercules AKUL/15/37_GOH1816N and FWO G.0929.15 to Pieter Vanden Berghe, University of Leuven.

\section{Funding}

This study was funded by grants to J.D.S (Fonds Wetenschappelijk Onderzoek $1533916 \mathrm{~N}$ ) and R.B. (Agentschap voor Innovatie door Wetenschap en Technologie SB-121393), IWT-140045 and HILIM-3D to C.M.V. and L.A.v.G, H2020-766181-DeLIVER-ITN to L.A.v.G., FWO-G0D9917N to C.M.V., and funding from the EU-ToxRisk, a project running under the European Union's Horizon 2020 research and innovation programme, grant agreement no. 681002.

\section{Author details}

'Department of Development and Regeneration, Stem Cell Institute, KU Leuven, Leuven, Belgium. ${ }^{2}$ Liver Cell Biology research group, Vrije Universiteit Brussel (VUB), Brussels, Belgium. ${ }^{3}$ The Massachusetts General Hospital Cancer Center, Harvard Medical School, Boston, MA 02114, USA. ${ }^{4}$ The Broad Institute of Harvard and MIT, Cambridge, MA 02142, USA. ${ }^{5}$ Electron Microscopy Platform of VIB Bio Imaging Core at KU Leuven and VIB-KU Leuven Center for Brain \& Disease Research, Leuven, Belgium

\section{Author contributions}

J.D.S. wrote all code for analysis and developed the CenTFinder package. J.D.S and I.T. analysed and interpreted the CenTFinder analysis, and performed the lentiviral transductions. J.D.S. and S.G. performed the RT-qPCR,

immunostainings, and cell culture. J.D.S., S.Z., B.T., S.G., and S.V. all contributed to (the setup of) the flow cytometry readings. Confocal imaging and colocalisation analysis was entirely performed by B.T., M.A.V., and R.B. generated the H9-ETV2 stem cell line, and performed initial characterisations. J. D.S. generated the H9-ETV2-SPI1 stem cell line. R.F.M.D.C. performed the computational image analysis on immunostainings and the $\lg G$ uptake assays. J.D.S. and P.B. performed the SEM preparation, and P.B. did the SEM imaging Primary mouse cells were isolated by E.A.v.O., FSA-FITC uptake assays were performed by E.A.v.O. and A.S., and IgG-AF555 uptake assays were performed by J.D.S., all statistical analyses were performed by J.D.S., J.D.S., L.A.V.G., and C.V. conceived the main concept of the presented study. J.D.S. and C.V. wrote majority of the manuscript. All authors contributed to the final version of the manuscript, as well as to discussions about and interpretations of the presented results.

Data availability

RNA-sequencing data is available from GEO (GSE156009).

Code availability

All code is reproducible and can be accessed via https://github.com/ jonathandesmedt92/CenTFinder.

Conflict of interest

The authors declare that they have no conflict of interest.

Ethics approval and consent to participate

No ethical approval was required for this study.

\section{Publisher's note}

Springer Nature remains neutral with regard to jurisdictional claims in published maps and institutional affiliations.

Supplementary Information accompanies this paper at (https://doi.org/ 10.1038/s41419-020-03356-2).

Received: 20 August 2020 Revised: 2 December 2020 Accepted: 7 December 2020

Published online: 14 January 2021

\section{References}

1. Fraser, R., Bosanquet, A. G. \& Day, W. A. Filtration of chylomicrons by the liver may influence cholesterol metabolism and atherosclerosis. Atherosclerosis $\mathbf{2 9}$, 113-123 (1978). 
2. Wisse, E., de Zanger, R. B., Charels, K., van der Smissen, P. \& McCuskey, R. S. The liver sieve: considerations concerning the structure and function of endothelial fenestrae, the sinusoidal wall and the space of disse. Hepatology $\mathbf{5}$, 683-692 (1985)

3. Smedsrød, B. et al. Hepatic sinusoidal cells in health and disease: update from the 14th International Symposium. Liver Int. 29, 490-501 (2009).

4. Sørensen, K. K. et al. The scavenger endothelial cell: a new player in homeostasis and immunity. Am. J. Physiol. Integr. Comp. Physiol. 303, R1217-R1230 (2012).

5. Ding, B. Sen et al. Inductive angiocrine signals from sinusoidal endothelium are required for liver regeneration. Nature 468, 310-315 (2010).

6. $\mathrm{Hu}$, J. et al. Endothelial cell-derived Angiopoietin-2 controls liver regeneration as a spatiotemporal rheostat. Science 343, 416-419 (2014).

7. Lai, W. K. et al. Expression of DC-SIGN and DC-SIGNR on human sinusoidal endothelium: a role for capturing hepatitis $C$ virus particles. Am. J. Pathol. 169 200-208 (2006)

8. Powlesland, A. S. et al. A novel mechanism for LSECtin binding to Ebola virus surface glycoprotein through truncated glycans. J. Biol. Chem. 283, 593-602 (2008).

9. Ganesan, L. P. et al. Rapid and efficient clearance of blood-borne virus by liver sinusoidal endothelium. PLoS Pathog 7, e1002281 (2011)

10. Simon-Santamaria, J. et al. Efficient uptake of blood-borne BK and JC polyomavirus-like particles in endothelial cells of liver sinusoids and renal Vasa recta. PLOS ONE 9, e111762 (2014).

11. Mates, J. M. et al. Mouse liver sinusoidal endothelium eliminates HIV-like particles from blood at a rate of 100 million per minute by a second-order kineticprocess. Front. Immunol 8, 35 (2017).

12. Steffan, A. M. et al. Primary cultures of endothelial cells from the human liver sinusoid are permissive for human immunodeficiency virus type 1. Proc. Natl Acad. Sci. USA 89, 1582-1586 (1992).

13. Boaru, S. G., Borkham-Kamphorst, E., Tihaa, L., Haas, U. \& Weiskirchen, R. Expression analysis of inflammasomes in experimental models of inflammatory and fibrotic liver disease. J. Inflamm. 9, 49 (2012).

14. Limmer, A. et al. Efficient presentation of exogenous antigen by liver endothelial cells to CD8+ T cells results in antigen-specific T-cell tolerance. Nat. Med. 6, 1348-1354 (2000).

15. Carambia, A. et al. TGF- $\beta$-dependent induction of CD4+CD25+Foxp3 + Tregs by liver sinusoidal endothelial cells. J. Hepatol. 61, 594-599 (2014).

16. Pasarín, M. et al. Sinusoidal endothelial dysfunction precedes inflammation and fibrosis in a model of NAFLD. PLOS ONE 7, e32785 (2012).

17. Xie, G. et al. Role of differentiation of liver sinusoidal endothelial cells in progression and regression of hepatic fibrosis in rats. Gastroenterology $\mathbf{1 4 2}$ 918 (2012).

18. DeLeve, L. D., Wang, X. \& Guo, Y. Sinusoidal endothelial cells prevent rat stellate cell activation and promote reversion to quiescence. Hepatology $\mathbf{4 8}$, 920-930 (2008).

19. Miyao, M. et al. Pivotal role of liver sinusoidal endothelial cells in NAFLD/NASH progression. Lab. Investig. 95, 1130-1144 (2015).

20. Rockey, D. C. \& Chung, J. J. Reduced nitric oxide production by endothelia cells in cirrhotic rat liver: endothelial dysfunction in portal hypertension. Gastroenterology 114, 344-351 (1998).

21. Elvevold, K., Nedredal, G. I., Revhaug, A., Bertheussen, K. \& Smesrød, B. Long term preservation of high endocytic activity in primary cultures of pig liver sinusoidal endothelial cells. Eur. J. Cell Biol. 84, 749-764 (2005)

22. Tokairin, $\mathrm{T}$. et al. A highly specific isolation of rat sinusoidal endothelial cells by the immunomagnetic bead method using SE-1 monoclonal antibody. J. Hepatol. 36, 725-733 (2002).

23. Krause, P. et al. Hepatocyte-supported serum-free culture of rat liver sinusoidal endothelial cells. J. Hepatol. 32, 718-726 (2000).

24. Tai, Y. et al. SK-Hep1: not hepatocellular carcinoma cells but a cell model for liver sinusoidal endothelial cells. Int. J. Clin. Exp. Pathol 11, 2931-2938 (2018).

25. Levenberg, S, Golub, J. S., Amit, M., Itskovitz-Eldor, J. \& Langer, R. Endothelia cells derived from human embryonic stem cells. Proc. Natl Acad. Sci. USA 99 4391-4396 (2002).

26. Sahara, M. et al. Manipulation of a VEGF-Notch signaling circuit drives formation of functional vascular endothelial progenitors from human pluripotent stem cells. Cell Res. 24, 820-841 (2014).

27. Elcheva, I. et al. Direct induction of haematoendothelial programs in human pluripotent stem cells by transcriptional regulators. Nat. Commun. 5, 1-11 (2014).
28. Koui, Y. et al. An in vitro human liver model by iPSC-derived parenchymal and non-parenchymal cells. Stem Cell Rep. 9, 490-498 (2017).

29. Gage, B. K. et al. Generation of functional liver sinusoidal endothelial cells from human pluripotent stem-cell-derived venous angioblasts. Cell Stem Cell $\mathbf{2 7}$ 254-269.e9 (2020)

30. Couvelard, A et al. Structural and functional differentiation of sinusoidal endothelial cells during liver organogenesis in humans. Blood 87, 4568-4580 (1996).

31. Gouysse, G. et al. Relationship between vascular development and vascular differentiation during liver organogenesis in humans. J. Hepatol. 37, 730-740 (2002).

32. Shiojiri, N. \& Sugiyama, Y. Immunolocalization of extracellular matrix components and integrins during mouse liver development. Hepatology 40, 346-355 (2004).

33. Zhang, $H$. et al. Genetic lineage tracing identifies endocardial origin of liver vasculature. Nat. Genet. 48, 537-543 (2016).

34. Tedesco, F. S. et al. Transplantation of genetically corrected human iPSCderived progenitors in mice with limb-girdle muscular dystrophy. Sci. Transl. Med 4, 140ra189 (2012)

35. Zhang, Y. et al. Rapid single-step induction of functional neurons from human pluripotent stem cells. Neuron 78, 785-798 (2013).

36. García-León, J. A. et al. SOX10 single transcription factor-based fast and efficient generation of oligodendrocytes from human pluripotent stem cells. Stem Cell Rep. 10, 655-672 (2018).

37. Boon, R. et al. Amino acid levels determine metabolism and CYP450 function of hepatocytes and hepatoma cell lines. Nat. Commun. 11, 1-16 (2020)

38. Langfelder, P. \& Horvath, S. WGCNA: an R package for weighted correlation network analysis. BMC Bioinformatics 9, 559 (2008).

39. Aibar, S. et al. SCENIC: single-cell regulatory network inference and clustering. Nat. Methods 14, 1083-1086 (2017).

40. Love, M. I., Huber, W. \& Anders, S. Moderated estimation of fold change and dispersion for RNA-seq data with DESeq2. Genome Biol. 15, 550 (2014).

41. Ordovás, L. et al. Efficient recombinase-mediated cassette exchange in hPSCs to study the hepatocyte lineage reveals AAVS1 locus-mediated transgene inhibition. Stem Cell Rep. 5, 918-931 (2015).

42. Aizarani, N. et al. A human liver cell atlas reveals heterogeneity and epithelial progenitors. Nature 572, 199-204 (2019).

43. Wagner, F., Barkley, D. \& Yanai, I. ENHANCE: accurate denoising of single-cell RNA-Seq data. Preprint at bioRxiv https://doi.org/10.1101/655365 (2019).

44. Scialdone, A. et al. Computational assignment of cell-cycle stage from singlecell transcriptome data. Methods 85, 54-61 (2015).

45. MacParland, S. A. et al. Single cell RNA sequencing of human liver reveals distinct intrahepatic macrophage populations. Nat. Commun. 9, 1-21 (2018).

46. Stradiot, L. et al. Functionality based method for simultaneous isolation of rodent hepatic sinusoidal cells. Biomaterials 139, 91-101 (2017).

47. Li, R. et al. Endocytosis of advanced glycation end-products in bovine choriocapillaris endothelial cells. Microcirculation 16, 640-655 (2009).

48. Lamprecht, M. R., Sabatini, D. M. \& Carpenter, A. E. CellProfilerTM: free, versatile software for automated biological image analysis. Biotechniques 42, 71-75 (2007).

49. Bolte, S. \& Cordelières, F. A guided tour into subcellular colocalization analysis in light microscopy. J. Microsc. 224, 213-232 (2006).

50. Schmid, C. D. et al. GPR182 is a novel marker for sinusoidal endothelial differentiation with distinct GPCR signaling activity in vitro. Biochem. Biophys. Res. Commun. 497, 32-38 (2018)

51. Ramachandran, $\mathrm{P}$. et al. Resolving the fibrotic niche of human liver cirrhosis at single-cell level. Nature 575, 512-518 (2019).

52. Hänzelmann, S., Castelo, R. \& Guinney, J. GSVA: gene set variation analysis for microarray and RNA-Seq data. BMC Bioinformatics 14, 7 (2013).

53. Koyano-Nakagawa, N. et al. Etv2 is expressed in the yolk sac hematopoietic and endothelial progenitors and regulates Lmo2 gene expression. Stem Cells 30, 1611-1623 (2012)

54. DeLeve, L. D., Wang, X., Hu, L., McCuskey, M. K. \& McCuskey, R. S. Rat liver sinusoidal endothelial cell phenotype is maintained by paracrine andautocrine regulation. Am. J. Physiol. Gastrointest. Liver Physio/ 287, G757-G763 (2004).

55. Elvevold, K, Smedsrød, B. \& Martinez, I. The liver sinusoidal endothelial cell: cell type of controversial and confusing identity. Am. J. Physiol. Gastrointest. Liver Physiol 294, G391-G400 (2007). 
56. Mistry, M., Gillis, J. \& Pavlidis, P. Meta-analysis of gene coexpression networks in the post-mortem prefrontal cortex of patients with schizophrenia and unaffected controls. BMC Neurosci. 14, 1-16 (2013).

57. Sun, J., Xu, H., Qi, M., Zhang, C. \& Jianxiang, S. H. I. Identification of key genes in osteosarcoma by meta-analysis of gene expression microarray. Mol. Med. Rep. 20, 3075-3084 (2019)

58. Triska, M., Ivliev, A., Nikolsky, Y. \& Tatarinova, T. V. Analysis of cis-regulatory elements in gene co-expression networks in cancer. Methods Mol. Biol. 1613 291-310 (2017)

59. Qian, J., Kluger, Y., Yu, H. \& Gerstein, M. Identification and correction of spurious spatial correlations in microarray data. Biotechniques 35, 42-48 (2003).

60. DeKoter, R. P. et al. Regulation of follicular B cell differentiation by the related E26 transformation-specific transcription factors PU.1, Spi-B, and Spi-C. J. Immunol. 185, 7374-7384 (2010).

61. Houston, I. B., Kamath, M. B., Schweitzer, B. L., Chlon, T. M. \& DeKoter, R. P. Reduction in PU.1 activity results in a block to B-cell development, abnormal myeloid proliferation, and neonatal lethality. Exp. Hematol. 35, 1056-1068 (2007).

62. Kamath, M. B. et al. Dose-dependent repression of T-cell and natural killer cell genes by PU.1 enforces myeloid and B-cell identity. Leukemia 22, 1214-1225 (2008).
63. Colucci, F. et al. Differential requirement for the transcription factor PU.1 in the generation of natural killer cells versus B and T cells. Blood 97, 2625-2632 (2001).

64. Danoy, M. et al. Transcriptome profiling of hiPSC-derived LSECs with nanoCAGE. Mol. Omics 16, 138-146 (2020).

65. Turkistany, S. A. \& Dekoter, R. P. The transcription factor PU.1 is a critical regulator of cellular communication in the immune system. Arch. Immunol. Ther. Exp. 59, 431-440 (2011)

66. Walter, T. J., Cast, A. E., Huppert, K. A. \& Huppert, S. S. Epithelial VEGF signaling is required in the mouse liver for proper sinusoid endothelial cell identity and hepatocyte zonation in vivo. Am. J. Physiol. Liver Physiol. 306, G849-G862 (2014).

67. Carpenter, B. et al. VEGF is crucial for the hepatic vascular development required for lipoprotein uptake. Development 132, 3293-3303 (2005).

68. Xie, G., Wang, L., Wang, X., Wang, L. \& DeLeve, L. D. Isolation of periportal, midlobular, and centrilobular rat liver sinusoidal endothelial cells enables study of zonated drug toxicity. Am. J. Physiol. Gastrointest. Liver Physiol. 299, G1204 (2010).

69. Wang, $X$. et al. Comparative analysis of cell lineage differentiation during hepatogenesis in humans and mice at the single-cell transcriptome level. Cell Res. 30, 1109-1126 (2020). 